\title{
Time Dependent Representations of the Stationary Wave Operators for "Oscillating" Long-Range Potentials
}

\author{
By \\ Kiyoshi MochizukI* and Jun UchiYama**
}

\section{Hntroduction}

Since the original paper of Dollard [7], the long-range scattering theory for the Schrödinger operators $-\Delta+V(x)$ has been studied by many authors (e.g., Buslaev-Matveev [5], Amrein-Martin-Misra [2], Alsholm-Kato [1], Hörmander [9], Kitada [13], Ikebe-Isozaki [10] and Kako [11]). These works treat the case that the potential $V(x)$ approaches zero without too much oscillation at infinity:

$$
\nabla^{\alpha} V(x)=0\left(r^{-|\alpha|-\delta}\right)(|\alpha|=0,1,2, \ldots) \text { for some } \delta>0
$$

$\left(\nabla=\nabla_{x}\right.$ is the gradient in $\mathbb{R}^{n}, r=|x|$ and $\alpha=\left(\alpha_{1}, \ldots, \alpha_{n}\right)$ are multi-indices with $\left.|\alpha|=\alpha_{1}+\cdots+\alpha_{n}\right)$, and prove the existence ([1], [2], [5], [9], [11]) and the completeness ([10], [13]) of the modified wave operators

$$
W_{D}^{ \pm}=s-\lim _{t \rightarrow \pm \infty} \exp \{i L t\} \exp \left\{-i L_{0} t-i X_{ \pm}(p, t)\right\} \quad \text { in } L^{2}\left(\mathbb{R}^{n}\right),
$$

where $L_{0}=-\Delta, L=-\Delta+V(x)$ on $L^{2}\left(\mathbb{R}^{n}\right), i=\sqrt{-1}, p=-i \nabla_{x}$ and $X_{ \pm}(\xi, t)$, $\xi \in \mathbb{R}^{n}$, solve the equations

$$
\partial_{t} X_{ \pm}(\xi, t)=V\left(2 \xi t+\nabla_{\xi} X_{ \pm}(\xi, t)\right) \quad\left(\partial_{t}=\partial / \partial t\right)
$$

near $t= \pm \infty$. The selfadjoint operators $X_{ \pm}(p, t)$ are called time dependent modifiers for $L$.

Stationary modifiers $Y_{ \pm}(x, \lambda), \lambda \in \mathbb{R}-\{0\}$, solve the equation

$$
\mp 2 \sqrt{\lambda} \partial_{r} Y_{ \pm}(x, \lambda)+\left|\nabla Y_{ \pm}(x, \lambda)\right|^{2}+V(x)=0\left(r^{-1-\delta}\right)
$$

Communicated by S. Matsuura, March 26, 1981.

* Department of Mathematics, Shinshu University, Matsumoto 390, Japan.

** Department of Mathematics, Kyoto University of Industrial Arts and Textile Fibres, Kyoto 606, Japan. 
near infinity. As we see in [10] and [13], $Y_{ \pm}(x, \lambda)$ can be obtained from $X_{ \pm}(\xi, t)$ by a kind of Legendre transformation in classical mechanics and are used to establish the completeness of $W_{\bar{D}}^{ \pm}$. On the other hand, $Y_{ \pm}(x, \lambda)$ are directly used in [11] to obtain another formulation of the modified wave operators. Let $\mathscr{E}_{0}(\lambda), \lambda \in \mathbb{R}$, be the spectral measure of $L_{0}$. Then in [11] is proved the following: For any pre-compact set $e \Subset(0, \infty)$, the limits

$$
W_{J}^{ \pm}(e)=s-\lim _{t \rightarrow \pm \infty} \exp \{i L t\} J_{ \pm}(e) \exp \left\{-i L_{0} t\right\} \mathscr{E}_{0}(e) \text { in } L^{2}\left(\mathbb{R}^{n}\right)
$$

exist, are isometry on $\mathscr{E}_{0}(e) L^{2}\left(\mathbb{R}^{n}\right)$ and coincide with $W_{D}^{ \pm} \mathscr{E}_{0}(e)$, where $J_{ \pm}(e)$ : $\mathscr{E}_{0}(e) L^{2}\left(\mathbb{R}^{n}\right) \rightarrow L^{2}\left(\mathbb{R}^{n}\right)$ are identification operators (cf. Kato [12]) defined by

$$
J_{ \pm}(e) f=(2 \pi)^{-n / 2} \int_{G(e)} \exp \left\{i x \cdot \xi-i Y_{ \pm}\left(x,|\xi|^{2}\right)\right\} \hat{f}(\xi) d \xi
$$

with $\hat{f}(\xi)$ being the Fourier transform of $f(x)$ and $G(e)=\left\{\xi ;|\xi|^{2} \in e\right\}$.

In this paper we shall partly extend the above mentioned results to a class of "oscillating", long-range potentials settled in our previous papers [14] and [15] (the definite conditions on $V(x)$ will be given in Section 1). Our main purpose is to show that modified wave opcrators of the form (0.5) exist and are complete for each $e \Subset\left(\Lambda_{\tilde{\delta}}, \infty\right)$, where the real number $\Lambda_{\tilde{\delta}}$ depends on the asymptotic conditions at infinity of $V(x)$. The results will be summarized in Theorem of Section 4.

Our "oscillating" long-range class includes the following examples:

$$
\begin{aligned}
& V(x)=c(x)+V_{s}(x), \\
& V(x)=\frac{c(x)}{\log r}+V_{s}(x) \quad(r=|x|), \\
& V(x)=c(x) \sin (\log r)+V_{s}(x), \\
& V(x)=\frac{\lambda(x) \sin \mu r}{r}+V_{s}(x),
\end{aligned}
$$

where $c(x)$ (real) satisfies the conditions

$$
c(x)=0(1), \nabla^{\alpha} c(x)=0\left(r^{-1-|\alpha| \delta}\right) \quad(|\alpha|=1,2,1 / 2<\delta<1)
$$

near infinity, $\lambda(x)$ (real) satisfies the conditions

$$
\nabla^{\alpha} \lambda(x)=0\left(r^{-|\alpha| \delta}\right) \quad(|\alpha|=0,1,2,1 / 2<\delta<1)
$$

near infinity, $\mu$ is a real number and $V_{s}(x)$ (real) is short-range, i.e., $V_{s}(x)$ $=0\left(r^{-1-\delta_{0}}\right)\left(\delta_{0}>0\right)$ near infinity. Note that (E.1) generalizes the usual $\frac{1}{2}$ long- 
range potential which satisfies (0.1) with $\delta>1 / 2$. Namely, by the terminology "oscillating" long-range potentials we never exclude ones which are in the frame of ordinary long-range potentials.

Now, in (0.5) we take $L_{0}=-\Delta+\Lambda_{\tilde{\delta}}$ on $L^{2}\left(\mathbb{R}^{n}\right)$. This choice of the free Hamiltonian mainly depends on the fact that we allow the case $\Lambda_{\tilde{\delta}}<0$. In fact, for the above examples $\Lambda_{\tilde{\delta}}$ is given by (cf. (1.2) and Assumption 2 of Section 1)

$$
\Lambda_{\tilde{\delta}}= \begin{cases}c_{\infty} & \text { for }(\mathbb{E} .1), \\ 0 & \text { for (E.2), } \\ \left|c_{\infty}\right| \sqrt{1+\varepsilon^{-2}} & \text { for (E.3), } \\ \lambda_{\infty}|\mu| / \varepsilon+\mu^{2} / 4 & \text { for (E.4), }\end{cases}
$$

where $c_{\infty}=\lim _{r \rightarrow \infty} c(x), \lambda_{\sigma}=\limsup _{r \rightarrow \infty} \frac{\lambda(x) \mu \cos \mu r}{|\mu|}$ and $\varepsilon=4 \min \left\{\delta_{0}, 2 \delta-1,1 / 2\right\}$, and so we have $\Lambda_{\tilde{\delta}}<0$ for (E.1) if $c_{\infty}<0$. Further, in our case, equation (0.4) does not work well, and it is necessary to define $J_{ \pm}(e)$ in a different manner. Let $\rho_{ \pm}(x, \lambda), \lambda>\Lambda_{\tilde{\delta}}$, be two solutions, specified in [15], of the equation

$$
\lambda_{r}^{2} \rho+\frac{n-1}{r} \partial_{r} \rho-\left(\partial_{r} \rho\right)^{2}+V(x)-\lambda=0\left(r^{-1-\delta}\right)
$$

near infinity. Then our identification operators $J_{ \pm}(e), e \Subset\left(A_{\bar{\delta}}, \infty\right)$, are defined by

$$
J_{ \pm}(e) f=\frac{ \pm 1}{2 i \sqrt{\pi}} \int_{e} \exp \left\{-\rho_{ \pm}(x, \lambda)\right\}\left[\mathscr{F}_{0} f\right](\lambda, \tilde{x}) d \lambda,
$$

where $\tilde{x}=x /|x|$ and $\mathscr{F}_{0}: L^{2}\left(\mathbb{P}^{n}\right) \rightarrow \mathbb{L}^{2}\left(\left(\Lambda_{\delta}, \infty\right) \times S^{n-1}\right)\left(S^{n-1}\right.$ being the unit sphere in $\left.\mathbb{R}^{n}\right)$ is a spectral representation of $L_{0}=-\Delta+\Lambda_{\tilde{\delta}}$ :

$$
\begin{aligned}
& {\left[\mathscr{F}_{0} f\right](\lambda, \tilde{x})=\frac{1}{\sqrt{2}}\left(\lambda-\Lambda_{\delta}\right)^{(n-2) / 4}} \\
& \quad \times(2 \pi)^{-n / 2} \int_{\mathbb{R}^{n}} \exp \left\{-i \sqrt{\lambda-\Lambda_{\delta}} \tilde{x} \cdot y-i \pi(n-3) / 4\right\} f(y) d y .
\end{aligned}
$$

We shall show that the operators $\exp \{i L t\} J_{ \pm}(e) \exp \left\{-i \mathbb{L}_{0} t\right\} \mathscr{E}_{0}(e)$ are bounded in $\mathscr{E}_{0}(e) L^{2}\left(\Re^{n}{ }^{n}\right)$ and strongly converge as $t \rightarrow \pm \infty$ to the stationary wave operators $U_{ \pm}(e)$. Here the existence and completeness of $U_{ \pm}(e)$ are already established in [15]. Our argument essentially bases on [14] and [15], whose results are summarized in Section 1. To show the boundedness of $J_{ \pm}(e)$ we shall follow a method of Calderón-Vaillancourt [6] on the $\mathbb{L}^{2}$-boundedness of pscudo-differential operators (Sections 2 and 3). On the other hand, for the 
proof of the convergence the stationary phase method will play an important role (Sections 4 and 5).

As we see in (0.7) for (E.3) and (E.4), $\left(\Lambda_{\tilde{\delta}}, \infty\right)$ does not in general cover the essential spectrum of $L=-\Delta+V(x)$. In this sense it remains some ambiguousness in our theory.

Here we note that potentials of the special form

$$
V(x)=V(r)=\frac{\lambda \sin \mu r^{\alpha}}{r^{\beta}}+V_{s}(r) \quad(\lambda, \mu \text { are real constants })
$$

including the case $\alpha=\beta=1$ (cf. (E.4)) have been studied by Dollard-Friedman [8], Ben-Artzi-Devinatz [4] and others. They reduce the problem to the study of ordinary differential operators on the half line $\boldsymbol{R}_{+}=(0, \infty)$, and prove the absolute continuity of the positive spectrum $(0, \infty)$ of $-\Delta+V(r)$ except for one possible eigenvalue $\mu^{2} / 4$, and the existence and completeness of the Møller wave operators. In this paper, we do not assume that $V(x)$ is spherically symmetric. However, our results for the concrete potential (0.11) with $\alpha=\beta=1$ (von Neumann-Wigner's adiabatic oscillator) is weaker than theirs. Also we have not shown whether or not our wave operators are equivalent to the ordinary Møller ones.

In case $V(x)=V_{s}(x)$, we can see that our modified wave operators coincide with the Møller wave operators modulo some simple unitary operators. Similar results can also be expected to the potential $V(x)$ which is improper integrable in $r=|x| \in \mathbb{R}_{+}$. It remains as an open problem so far.

\section{§1. Assumptions and Preliminaries}

Let $\Omega$ be an infinite domain in $\boldsymbol{R}^{n}$ with smooth compact boundary $\partial \Omega$ lying inside some sphere $S\left(R_{0}\right)=\left\{x ;|x|=R_{0}\right\}$. We consider in $\Omega$ the Schrödinger operator $-\Delta+V(x)$, where $\Delta$ is the Laplacian and $V(x)$ is a potential function. We assume

Assumption 1. $V(x)=V_{1}(x)+V_{s}(x)$, where $V_{1}(x)$ is a real-valued function satisfying the "Stummel condition" for some $\mu>0$ :

$$
\begin{cases}\sup _{x \in \Omega} \int_{|x-y|<1}\left|V_{1}(y)\right|^{2}|x-y|^{-n+4-\mu} d y<\infty & \text { (if } n \geq 4), \\ \sup _{x \in \Omega} \int_{|x-y|<1}\left|V_{1}(y)\right|^{2} d y<\infty & \text { (if } n \leq 3),\end{cases}
$$


and $V_{s}(x)$ is a real-valued bounded measurable function in $\Omega$. Moreover, the unique continuation property holds for both $-\Delta+V(x)$ and $-\Delta+V_{1}(x)$.

Assumption 2. $V_{1}(x)$ is an "oscillating" long-range potential; that is, there exist some constants $C_{1}>0, R_{1} \geq R_{0}, a \geq 0$ and $1 / 2<\delta_{j}<1(j=1,2)$ such that for any $x \in B\left(R_{1}\right)=\left\{x ;|x|>R_{1}\right\}$,

(i) $\left|V_{1}(x)\right| \leq C_{1}$,

(ii) $\left|\partial_{r} V_{1}(x)\right| \leq C_{1} r^{-1}$,

(iii) $\left|\partial_{r}^{2} V_{1}(x)+a V_{1}(x)\right| \leq C_{1} r^{-1-\delta_{1}}$,

(iv) $\left|\left(\nabla-\tilde{x} \partial_{r}\right) V_{1}(x)\right| \leq C_{1} r^{-1-\delta_{2}}$,

(v) $\left|\left(\nabla-\tilde{x} \partial_{r}\right) \partial_{r} V_{1}(x)\right| \leq C_{1} r^{-1-\delta_{1}}$,

(vi) $\left|\left(\nabla-\tilde{x} \partial_{r}\right) \cdot\left(\nabla-\tilde{x} \partial_{r}\right) V_{1}(x)\right| \leq C_{1} r^{-1-2 \delta_{2}}$.

On the other hand, $V_{s}(x)$ is a short-range potential; that is, there exist some constants $C_{2}>0$ and $0<\delta_{0}<1$ such that for any $x \in B\left(R_{1}\right)$,

(vii) $\left|V_{s}(x)\right| \leq C_{2} r^{-1-\delta_{0}}$.

In the following we put $\delta=\min \left\{\delta_{0}, \delta_{1}, \delta_{2}\right\}$ and $\tilde{\delta}=\min \left\{\delta, 2 \delta_{2}-1\right\}$. Note that the condition $\delta_{j}<1(j=0,1,2)$ does not restrict the generality.

We put

$$
E(\gamma)=\limsup _{r \rightarrow \infty} \frac{1}{\gamma}\left\{r \partial_{r} V_{1}(x)+\gamma V_{1}(x)\right\} \quad \text { for } \quad \gamma>0,
$$

and define $\Lambda_{\sigma}, \sigma>0$, as follows:

$$
\Lambda_{\sigma}=E(\min \{4 \sigma, 2\})+a / 4,
$$

where $a \geq 0$ is the constant given in (iii) of Assumption 2. Then as is discussed in $[14 ; \S 8]$, we have the

Lemma 1.1. $\Lambda_{\sigma}$ is non-increasing and continuous in $\sigma>0$, and

$$
\Lambda_{1 / 2}=\min _{\sigma>0} \Lambda_{\sigma} \geq \limsup _{r \rightarrow \infty} V_{1}(x)+a / 4,
$$

where

$$
\left|V_{1}(x)\right| \leq C_{3} r^{-1} \quad \text { in } B\left(R_{1}\right) \quad \text { if } a>0 \text {. }
$$

We put

$$
\eta(\lambda)=4 \lambda /(4 \lambda-a) \text { for } \lambda>\Lambda_{1 / 2} .
$$

Note that $\eta(\lambda) \equiv 1$ if $a=0$. Then by means of (1.3) and (1.4) we can easily prove the following 
Lemma 1.2. Let $\varepsilon$ be any constant satisfying $0<\varepsilon<1$. Then there exist some constants $C_{4}>0$ and $R_{1}^{\prime} \geq R_{1}$ depending only on $\varepsilon$ such that for any $(x, \lambda) \in B\left(R_{1}^{\prime}\right) \times\left[\Lambda_{1 / 2}+\varepsilon, \infty\right)$,

$$
\begin{array}{ll}
\varepsilon / 2 \leq \lambda-\eta(\lambda) V_{1}(x) \leq|\lambda|+C_{4}, & \\
1 / 2 \leq \partial_{\lambda}\left\{\lambda-\eta(\lambda) V_{1}(x)\right\} \leq 2 & \left(\partial_{\lambda}=\partial / \partial \lambda\right), \\
\left|\partial_{\lambda}^{l}\left\{\lambda-\eta(\lambda) V_{1}(x)\right\}\right| \leq C_{4} r^{-1} & (l=2 \sim 6) .
\end{array}
$$

For some $R_{2} \geq R_{1}^{\prime}$ and any $(x, \lambda) \in B\left(R_{2}\right) \times\left[\Lambda_{1 / 2}+\varepsilon, \infty\right)$ we put

$$
\begin{aligned}
& \rho_{ \pm}(x, \lambda) \\
& \quad=\mp i \int_{R_{2}}^{r} \sqrt{\lambda-\eta(\lambda) V_{1}(s \tilde{x})} d s+\frac{n-1}{2} \log r+\frac{1}{4} \log \left\{\lambda-\eta(\lambda) V_{1}(x)\right\} .
\end{aligned}
$$

Then by a straight calculation (cf. Lemma 1.1 of [15]) we have the

Lemma 1.3. There exists a constant $C_{5}>0$ depending on $\varepsilon$ such that for any $(x, \lambda) \in B\left(R_{2}\right) \times\left[\Lambda_{1 / 2}+\varepsilon, \infty\right)$,

$$
\begin{aligned}
& \left|\partial_{r}^{2} \rho_{ \pm}+\frac{n-1}{r} \partial_{r} \rho_{ \pm}-\left(\partial_{r} \rho_{ \pm}\right)^{2}+V_{1}(x)-\lambda\right| \leq C_{5} r^{-1-\delta_{1}}, \\
& \left|\left(\nabla-\tilde{x} \partial_{r}\right) \rho_{ \pm}\right| \leq C_{5} r^{-\delta_{2}}, \\
& \left|\left(\nabla-\tilde{x} \partial_{r}\right) \partial_{r} \rho_{ \pm}\right| \leq C_{5} r^{-1-\min \left\{\delta_{1}, \delta_{2}\right\}}, \\
& \left|\left(\nabla-\tilde{x} \partial_{r}\right) \cdot\left(\nabla-\tilde{x} \partial_{r}\right) \rho_{ \pm}\right| \leq C_{5} r^{-2 \delta_{2}} .
\end{aligned}
$$

For any real number $\mu$ and $G \subset \Omega$, let $L_{\mu}^{2}(G)$ denote the space of all functions $f(x)$ such that

$$
\|f\|_{\mu, G}^{2}=\int_{G}(1+r)^{2 \mu}|f(x)|^{2} d x<\infty .
$$

If $\mu=0$ or $G=\Omega$, the subscript $\mu$ or $G$ will be omitted. Let $\alpha, \beta$ be a pair of positive constants satisfying

$$
0<\alpha \leq \beta \leq 1 \quad \text { and } \quad \alpha+\beta \leq 2 \delta .
$$

For $\lambda>\Lambda_{\beta / 2}\left(\geq \Lambda_{\delta}\right)$ and $f \in L_{(1+\beta) / 2}^{2}(\Omega)$ let us consider the exterior boundaryvalue problem

$$
\left\{\begin{array}{l}
(-\Delta+V(x)-\lambda) u(x)=f(x) \text { in } \Omega \\
B u=\left\{\begin{array}{l}
\text { or } v(x) \cdot \nabla u+d(x) u \\
v \text { on } \partial \Omega,
\end{array}\right.
\end{array}\right.
$$

where $v(x)=\left(v_{1}(x), \ldots, v_{n}(x)\right)$ is the outer unit normal to the boundary $\partial \Omega$ and 
$d(x)$ is a real-valued smooth function on $\partial \Omega$. The outgoing $(+)$ or incoming (-) solution of (1.16) will be distinguished by the radiation condition

$(1.17)_{ \pm} \quad u \in L_{-(1+u) / 2}^{2}(\Omega)$ and $\partial_{r} u+\left(\partial_{r} \rho_{ \pm}(x, \lambda)\right) u \in L_{(-1+\beta) / 2}^{2}\left(B\left(R_{2}\right)\right)$

Now let $L$ be the selfadjoint operator in $L^{2}(\Omega)$ defined by

$$
\left\{\begin{array}{l}
\mathscr{D}(L)=\left\{u \in L^{2}(\Omega) ; \Delta u \in L^{2}(\Omega) \quad \text { and } B u=0 \quad \text { on } \partial \Omega\right\} \\
L u=-\Delta u+V(x) u \quad \text { for } u \in \mathscr{D}(L),
\end{array}\right.
$$

and let $R(\zeta)(\zeta \in \mathbb{C}-\mathbb{R})$ and $\mathscr{E}(\lambda)(\lambda \in \mathbb{R})$ be its resolvent and spectral measure, respectively. Then the main results of [14] and [15] can be summarized in the following propositions. To show Proposition 1.1 we require (1.10) and (1.12) (see Theorems $1-5$ of [14]). To show Proposition 1.2 we require (1.10) and (1.11) (see Theorem 2.1 of [15]). (1.13) is used to show Proposition 1.3 (see Theorems 3.1, 4.1 and 6.1 of [15]).

Proposition 1.1. (a) Let $\alpha, \beta$ be any pair satisfying (1.15), and let $\varepsilon$ and $N$ be any constants satisfying $0<\varepsilon<1<N<\infty$. Then there exists a constant $C_{6}>0$ such that for any $f \in L_{(1+\beta) / 2}^{2}(\Omega)$ (which is dense in $L^{2}(\Omega)$ ), $\lambda \in\left[\Lambda_{\beta / 2}+\varepsilon\right.$, $\left.\Lambda_{\beta / 2}+N\right]$ and $\tau \in(0,1)$,

$$
\|R(\lambda \pm i \tau) f\|_{-(1+\sigma) / 2} \leq C_{6}\|f\|_{(1+\beta) / 2} .
$$

Moreover, $R(\lambda \pm i \tau) f$ converges in $L_{-(1+\alpha) / 2}^{2}(\Omega)$ to the unique outgoing [or incoming] solution $u_{ \pm}=R_{ \pm}(\lambda) f$ of $(1.16)$ as $\tau \downarrow 0$.

(b) The above convergence is uniform in $\lambda \in\left[\Lambda_{\beta / 2}+\varepsilon, \Lambda_{\beta / 2}+N\right]$. Thus, $R_{ \pm}(\lambda) f$ is continuous in $L_{-(1+\alpha) / 2}^{2}(\Omega)$ with respect to $(\lambda, f) \in\left(\Lambda_{\beta / 2}, \infty\right)$ $\times L_{(1+\beta) / 2}^{2}(\Omega)$.

(c) Let $R_{ \pm}^{*}(\lambda): L_{(1+\alpha) / 2}^{2}(\Omega) \rightarrow L_{-(1+\beta) / 2}^{2}(\Omega)$ be the adjoint of $R_{ \pm}(\lambda)$. Then we have for any' $f \in L_{(1+\beta) / 2}^{2}(\Omega)\left(\subset L_{(1+\gamma) / 2}^{2}(\Omega)\right)$ and $\lambda \in\left(\Lambda_{\beta / 2}, \infty\right)$,

$$
R_{ \pm}^{*}(\lambda) f=R_{\mp}(\lambda) f .
$$

(d) For any pre-compact set $e \Subset\left(\Lambda_{\beta / 2}, \infty\right)$ and $f, g \in L_{(1+\beta) / 2}^{2}(\Omega)$ we have

$$
(\mathscr{E}(e) f, g)=(2 \pi i)^{-1} \int_{\epsilon}\left(R_{+}(\lambda) f-R_{-}(\lambda) f, g\right) d \lambda,
$$

where (, ) denotes the inner product in $L^{2}(\Omega)$, or more generally, the duality between $L_{-(1+\alpha) / 2}^{2}(\Omega)$ and $L_{(1+\alpha) / 2}^{2}(\Omega)$. Thus, the part of $L$ in $\mathscr{E}\left(\left(\Lambda_{\delta}, \infty\right)\right) L^{2}(\Omega)$ is absolutely continuous with respect to the Lebesgue measure on $\lambda \in\left(\Lambda_{\delta}, \infty\right)$. 
Remark 1.1. Let $R_{1, \pm}(\lambda)$ be the operator $R_{ \pm}(\lambda)$ with $V_{s}(x) \equiv 0$. In this case we can choose $\beta=1$ in (1.15), where $\alpha$ should be chosen as

$$
0<\alpha \leq 2 \min \left\{\delta_{1}, \delta_{2}\right\}-1 .
$$

Proposition 1.2. (a) For any $\alpha, \beta$ satisfying (1.15), let $f \in L_{(1+\beta) / 2}^{2}(\Omega)$ and $\lambda \geq \Lambda_{\beta / 2}+\varepsilon$. Then there exists a sequence $r_{l}=r_{l}(\alpha, \beta, f, \lambda)$ diverging to $\infty$ such that

$$
\lim _{l \rightarrow \infty} \int_{S\left(r_{l}\right)}\left\{r^{-\alpha}\left|R_{1, \pm}(\lambda) f\right|^{2}+r^{\beta}\left|\left(\nabla+\tilde{x} \partial_{r} \rho_{ \pm}\right) R_{1, \pm}(\lambda) f\right|^{2}\right\} d S=0 .
$$

(b) For any $\lambda \in\left[\Lambda_{1 / 2}+\varepsilon, \infty\right)$ and $f \in L_{1}^{2}(\Omega)$, let $r_{l}=r_{l}(\alpha, 1, f, \lambda)$, where $\alpha$ satisfies (1.22). Then

$$
\mathscr{F}_{1, \pm}\left(\lambda, r_{l}\right) f \equiv \frac{1}{\sqrt{\pi}} \exp \left\{\rho_{ \pm}\left(r_{l} \cdot, \lambda\right)\right\}\left[R_{1, \pm}(\lambda) f\right]\left(r_{l} \cdot\right)
$$

strongly converges in $L^{2}\left(S^{n-1}\right)$ as $l \rightarrow \infty$. Let $\mathscr{F}_{1, \pm}(\lambda): L_{1}^{2}(\Omega) \rightarrow L^{2}\left(S^{n-1}\right)$ be defined by

$$
\mathscr{F}_{1, \pm}(\lambda) f=s-\lim _{l \rightarrow \infty} \mathscr{F}_{1, \pm}\left(\lambda, r_{l}\right) f \quad \text { in } \quad L^{2}\left(S^{n-1}\right)
$$

Then we have

$$
\left\|\mathscr{F}_{1, \pm}(\lambda) f\right\|_{L^{2}\left(S^{n-1}\right)}^{2}=(2 \pi i)^{-1}\left(R_{1,+}(\lambda) f-R_{1,-}(\lambda) f, f\right) .
$$

Moreover, $\mathscr{F}_{1, \pm}(\lambda)$ is independent of the choice of $r_{l}$.

(c) Let $\tilde{\alpha}, \tilde{\beta}$ satisfy

$$
0<\tilde{\alpha} \leq \tilde{\beta} \leq 1 \quad \text { and } \quad \tilde{\alpha}+\tilde{\beta} \leq 2 \tilde{\delta}=\min \left\{2 \delta, 4 \delta_{2}-2\right\}
$$

((1.27) is a stronger condition than (1.15)). Then for any $\lambda \in\left[\Lambda_{\tilde{\beta} / 2}+\varepsilon, \infty\right)$ the operator $\mathscr{F}_{1, \pm}(\lambda)$ can be extended to a bounded operator from $L_{(1+\tilde{\beta}) / 2}^{2}(\Omega)$ to $L^{2}\left(S^{n-1}\right)$ by continuity. Denoting the extended operator by $\mathscr{F}_{1, \pm}(\lambda)$ again, we have for any $f \in L_{(1+\tilde{\beta}) / 2}^{2}(\Omega), \phi \in L^{2}\left(S^{n-1}\right)$ and $\lambda \in\left[\Lambda_{\tilde{\beta} / 2}+\varepsilon, \infty\right)$,

$$
\left(\mathscr{F}_{1, \pm}(\lambda) f, \phi\right)_{L^{2}\left(S^{n-1}\right)}=\lim _{l \rightarrow \infty}\left(\mathscr{F}_{1, \pm}\left(\lambda, r_{l}\right) f, \phi\right)_{L^{2}\left(S^{n-1}\right)},
$$

where $r_{l}=r_{l}(\tilde{\alpha}, \tilde{\beta}, f, \lambda)$.

Remark 1.2. In [15] we neglect the fact that $R_{1}^{\prime}$ in Lemma 1.2 depends on $\varepsilon$, and then $\mathscr{F}_{1: \pm}(\lambda)$ depends on $\varepsilon$ and $R_{2} \geq R_{1}^{\prime}$ by (1.9) and (1.25). So the above and the following propositions are corrections of [15]. Let $\varepsilon^{\prime}$ and $R_{1}^{\prime \prime}$ be another pair and let $\mathscr{F}_{1 . \pm}^{\prime}(\lambda)$ be the operator $\mathscr{F}_{1, \pm}(\lambda)$ corresponding to $\varepsilon^{\prime}$ and some $R_{2}^{\prime} \geq R_{1}^{\prime \prime}$. Then for $\lambda \in\left[\Lambda_{\tilde{\beta} / 2}+\varepsilon, \infty\right) \cap\left[\Lambda_{\tilde{\beta} / 2}+\varepsilon^{\prime}, \infty\right), \mathscr{F}_{1, \pm}^{\prime}(\lambda)$ coincides with 
$\mathscr{F}_{1, \pm}(\lambda)$ modulo a unitary operator on $L^{2}\left(S^{n-1}\right)$ :

$$
\left[\mathscr{F}_{1, \pm}^{\prime}(\lambda) f\right](\tilde{x})=\exp \left\{ \pm i \int_{R_{2}}^{R_{2}^{\prime}} \sqrt{\lambda-\eta(\lambda) V_{1}(s \tilde{x})} d s\right\}\left[\mathscr{F}_{1, \pm}(\lambda) f\right](\tilde{x})
$$

for $f \in L_{(1+\tilde{\beta}) / 2}^{2}(\Omega)$.

Remark 1.3. Let $\mathscr{F}_{0}(\lambda)$ be the operator $\mathscr{F}_{1,+}(\lambda)$ corresponding to the selfadjoint operator $L_{0}=-\Delta+\Lambda_{\tilde{\delta}}$ on $L^{2}\left(\mathbb{R}^{n}\right)$. In this case, $\eta(\lambda) V_{1}(x) \equiv \Lambda_{\tilde{\delta}}$ being constant, we can choose $R_{2}=0$. Then for any $\lambda>\Lambda_{\tilde{\delta}}, \mathscr{F}_{0}(\lambda) f$ is represented by the right side of $(0.10)$ (see Remark 6.2 of [15]).

Proposition 1.3. Let $\tilde{\alpha}, \tilde{\beta}$ satisfy (1.27) and $\Lambda_{\tilde{\delta}} \leq \Lambda_{\tilde{\beta} / 2}<\Lambda_{\tilde{\delta}}+\varepsilon$.

(a) For $\lambda \in\left[\Lambda_{\tilde{\beta} / 2}+\varepsilon, \infty\right)$ let

$$
\mathscr{F}_{ \pm}(\lambda)=\mathscr{F}_{1, \pm}(\lambda)\left\{1-V_{s} R_{ \pm}(\lambda)\right\} .
$$

Then it defines a bounded operator from $L_{(1+\tilde{\beta}) / 2}^{2}(\Omega)$ to $L^{2}\left(S^{n-1}\right)$. Moreover, it depends continuously on $\lambda$.

(b) Let $\mathscr{F}_{ \pm}: L_{(1+2 \delta) / 2}^{2}(\Omega) \rightarrow L^{2}\left(\left[\Lambda_{\tilde{\delta}}+2 \varepsilon, \infty\right) \times S^{n-1}\right)$ be defined $b y$

$$
\left[\mathscr{F}_{ \pm} f\right](\lambda, \tilde{x})=\left[\mathscr{F}_{ \pm}(\lambda) f\right](\tilde{x}),(\lambda, \tilde{x}) \in\left[\Lambda_{\tilde{\delta}}+2 \varepsilon, \infty\right) \times S^{n-1} .
$$

Then $\mathscr{F}_{ \pm}$can be extended to a partial isometric operator from $L^{2}(\Omega)$ onto $L^{2}\left(\left[\Lambda_{\tilde{\delta}}+2 \varepsilon, \infty\right) \times S^{n-1}\right)$ with initial set $\mathscr{E}\left(\left[\Lambda_{\tilde{\delta}}+2 \varepsilon, \infty\right)\right) L^{2}(\Omega)$. The extended operator will be denoted by $\mathscr{F}_{ \pm}$again.

(c) (Spectral representations) For any bounded Borel function $b(t)$ on $\mathbb{R}$ and any $f \in L^{2}(\Omega)$, we have

$$
\begin{aligned}
& \mathscr{E}( {\left.\left[\Lambda_{\tilde{\delta}}+2 \varepsilon, \infty\right)\right) b(L) f=\mathscr{F}_{ \pm}^{*} b(\lambda) \mathscr{F}_{ \pm} f } \\
& \quad=s-\lim _{N \rightarrow \infty} \int_{\Lambda_{\tilde{\delta}}^{+2 \varepsilon}}^{N} \mathscr{F}_{ \pm}^{*}(\lambda) b(\lambda)\left[\mathscr{F}_{ \pm} f\right](\lambda, \cdot) d \lambda \quad \text { in } \quad L^{2}(\Omega),
\end{aligned}
$$

where $\mathscr{F}_{ \pm}^{*}: L^{2}\left(\left[\Lambda_{\tilde{\delta}}+2 \varepsilon, \infty\right) \times S^{n-1}\right) \rightarrow L^{2}(\Omega)$ is the adjoint of $\mathscr{F}_{ \pm}$and $\mathscr{F}_{ \pm}^{*}(\lambda)$ : $L^{2}\left(S^{n-1}\right) \rightarrow L_{-(1+\tilde{\beta}) / 2}^{2}(\Omega)$ is the adjoint of $\mathscr{F}_{ \pm}(\lambda)$.

(d) (Stationary wave operators) We put for any pre-compact set $e$ c $\left[\Lambda_{\tilde{\delta}}+2 \varepsilon, \infty\right)$,

$$
U_{ \pm}(e)=\mathscr{F}_{ \pm}^{*} \mathscr{F}_{0} \mathscr{E}_{0}(e)
$$

where $\mathscr{E}_{0}(\lambda), \lambda \in \mathbb{R}$, is the spectral measure of $L_{0}$. Then each $U_{ \pm}(e)$ is a unitary operator from $\mathscr{E}_{0}(e) L^{2}\left(\mathbb{R}^{n}\right)$ onto $\mathscr{E}(e) L^{2}(\Omega)$, which intertwines the operators $\mathscr{E}_{0}(e) L_{0}$ and $\mathscr{E}(e) L$. Namely, we have for any bounded Borel function $b(t)$ on $\mathbb{R}$, 


$$
\begin{aligned}
& \mathscr{E}(e) b(L)=U_{ \pm}(e) \mathscr{E}_{0}(e) b\left(L_{0}\right) U_{ \pm}^{*}(e), \\
& \mathscr{E}_{0}(e) b\left(L_{0}\right)=U_{ \pm}^{*}(e) \mathscr{E}(e) b(L) U_{ \pm}(e),
\end{aligned}
$$

where $U_{ \pm}^{*}(e): \mathscr{E}(e) L^{2}(\Omega) \rightarrow \mathscr{E}_{0}(e) L^{2}\left(\mathbb{R}^{n}\right)$ is the adjoint of $U_{ \pm}(e)$.

\section{§2. Expressions of $\mathscr{F}_{ \pm}^{*}(\mathcal{Z})$ and the Identification Operators $\mathbb{I}_{ \pm}(e)$}

Let $\rho_{ \pm}$be as given in (1.9) with some $R_{2} \geq R_{1}^{\prime}$ and $e=\left(\lambda_{1}, \lambda_{2}\right)$ be a bounded interval in $\left[\Lambda_{\tilde{\delta}}+2 \varepsilon, \infty\right)$. For any $\phi(\lambda, \tilde{x}) \in C_{0}^{\infty}\left(e \times S^{n-1}\right)$ we put

$$
\begin{aligned}
v_{\phi, \pm}(x, \lambda)= \begin{cases}\frac{1}{\sqrt{\pi}} \exp \left\{-\rho_{ \pm}(x, \lambda)\right\} \phi(\lambda, \tilde{x}) \psi(r), & |x|=r>R_{2}+1 \\
0, & |x|=r<R_{2}+1\end{cases} \\
g_{\phi, \pm}(x, \lambda)=\{-\Delta+V(x)-\lambda\} v_{\phi, \pm}(x, \lambda),
\end{aligned}
$$

where $\psi(r)$ is a smooth function of $r>0$ such that $0 \leq \psi(r) \leq 1, \psi(r)=0$ for $r$ $<R_{2}+1$ and $=1$ for $r>R_{2}+2$. Note that

$$
\begin{aligned}
g_{\phi, \pm}= & \frac{1}{\sqrt{\pi}} \exp \left\{-\rho_{ \pm}\right\}\left[\left\{\partial_{r}^{2} \rho_{ \pm}+\frac{n-1}{r} \partial_{r} \rho_{ \pm}-\left(\partial_{r} \rho_{ \pm}\right)^{2}+V^{\prime}-\lambda\right\} \phi \psi\right. \\
& +\left\{\left(\nabla-\tilde{x} \partial_{r}\right) \cdot\left(\nabla-\tilde{x} \partial_{r}\right) \rho_{ \pm}-\left(\left(\nabla-\tilde{x} \partial_{r}\right) \rho_{ \pm}\right)^{2}\right\} \phi \psi-\left\{\psi^{\prime \prime}+\frac{n-1}{r} \psi^{\prime}\right. \\
& \left.\left.-2\left(\partial_{r} \rho_{ \pm}\right) \psi^{\prime}\right\} \phi-\left\{\left(\nabla-\tilde{x} \partial_{r}\right) \cdot\left(\nabla-\tilde{x} \partial_{r}\right) \phi-2\left(\nabla-\tilde{x} \partial_{r}\right) \rho_{ \pm} \cdot \nabla \phi\right\} \psi\right]
\end{aligned}
$$

Here $\left(\nabla-\tilde{x} \partial_{r}\right) \cdot\left(\nabla-\tilde{x} \partial_{r}\right) \phi=0\left(r^{-2}\right)$ and $\nabla \phi=0\left(r^{-1}\right)$ near infinity. Then as is easily seen from (1.9), (vii) and Lemma 1.3, we have

Lemma 2.1. There exists a constant $C_{7}>0$ such that for any $(x, \lambda)$ $\in B\left(R_{2}+1\right) \times e$,

$$
\begin{aligned}
& \left|g_{\phi, \pm}(x, \lambda)\right| \leq C_{7} r^{-(n-1) / 2} r^{-(1+\tilde{\delta})} \\
& \left|v_{\phi, \pm}(x, \lambda)\right| \leq C_{7} r^{-(n-1) / 2} .
\end{aligned}
$$

Moreover, we have

$$
\begin{gathered}
\left\{\partial_{r}+\partial_{r} \rho_{ \pm}(x, \lambda)\right\} v_{\phi, \pm}(x, \lambda)=0 \quad \text { in } \quad(x, \lambda) \in B\left(R_{2}+2\right) \times e, \\
B v_{\phi, \pm}(x, \lambda)=0 \quad \text { on } \quad(x, \lambda) \in \partial \Omega \times e .
\end{gathered}
$$

Let $\tilde{\alpha}, \tilde{\beta}$ be as given in Proposition 1.3. Then (2.4) implies that $g_{\phi, \pm}$ $\in L_{(1+\tilde{\beta}) / 2}^{2}(\Omega)$, and it follows from (2.5)-(2.7) that $v_{\phi, \pm}$ determines an outgoing [incoming] solution of (1.16) and (1.17) \pm with $f=g_{\phi, \pm}, \alpha=\tilde{\alpha}$ and $\beta=\tilde{\beta}$. Namely, we have 


$$
v_{\phi, \pm}(\cdot, \lambda)=\left[R_{ \pm}(\lambda) g_{\phi, \pm}(\cdot, \lambda)\right](\cdot), \lambda \in e .
$$

Proposition 2.1. For any $\phi(\lambda, \tilde{x}) \in C_{0}^{\infty}\left(e \times S^{n-1}\right), \mathscr{F}_{ \pm}^{*}(\lambda) \phi(\lambda, \cdot) \in L_{-(1+\tilde{\beta}) / 2}^{2}(\Omega)$ is expressed as follows:

$$
\left[\mathscr{F}_{ \pm}^{*}(\lambda) \phi(\lambda, \cdot)\right](x)=\frac{ \pm 1}{2 i}\left\{v_{\phi, \pm}(x, \lambda)-\left[R_{\mp}(\lambda) g_{\phi,=}(\cdot, \lambda)\right](x)\right\} .
$$

Proof. For $f \in L_{(1+\tilde{\beta}) / 2}^{2}(\Omega)$ and $\lambda \in e$ let

$$
u_{ \pm}(\cdot, \lambda)=R_{ \pm}(\lambda) f=R_{1, \pm}(\lambda)\left\{1-V_{s} R_{ \pm}(\lambda)\right\} f .
$$

Noting $\left\{1-V_{s} R_{ \pm}(\lambda)\right\} f \in L_{(1+\tilde{\beta}) / 2}^{2}(\Omega)$, we choose a sequence $r_{l}=r_{l}(\tilde{\alpha}, \tilde{\beta},\{1$ $\left.\left.-V_{s} R_{ \pm}(\lambda)\right\} f, \lambda\right)$ diverging to $\propto$ as in Proposition $1.2(\mathrm{a})$. Let $\Omega\left(r_{l}\right)=\{x \in \Omega$; $\left.|x|<r_{l}\right\}$. Then by the Green formula, (2.6) and (2.7) we have

$$
\begin{aligned}
& \frac{ \pm 1}{2 i} \int_{\Omega\left(r_{l}\right)}\left\{u_{ \pm} \overline{g_{\phi, \pm}}-f \overline{v_{\phi, \pm}}\right\} d x \\
& \quad=\frac{ \pm 1}{2 i} \int_{S\left(r_{l}\right)}\left\{\partial_{r} u_{ \pm} \overline{v_{\phi, \pm}}-u_{ \pm} \partial_{r} \overline{v_{\phi, \pm}}\right\} d S \\
& \quad=\frac{ \pm 1}{2 i} \int_{S\left(r_{l}\right)}\left(\partial_{r}+\partial_{r} \rho_{ \pm}\right) u_{ \pm} \overline{v_{\phi, \pm}} d S \mp \int_{S\left(r_{l}\right)}\left(\operatorname{Im} \partial_{r} \rho_{ \pm}\right) u_{ \pm} \overline{v_{\phi, \pm}} d S
\end{aligned}
$$

Here by (1.9) and (2.1),

$$
\begin{aligned}
& \mp \int_{S\left(r_{l}\right)}\left(\operatorname{Im} \hat{c}_{r} \rho_{ \pm}\right) u_{ \pm} \overline{v_{\phi, \pm}} d S=\int_{S\left(r_{l}\right)} \sqrt{\lambda-\eta(\lambda) V_{1}(x)} u_{ \pm} \overline{v_{\phi, \pm}} d S \\
& \quad=\int_{S^{n-1}} \frac{1}{\sqrt{\pi}} \exp \left\{\rho_{ \pm}\left(\ddot{r}_{l}, \tilde{x}, \lambda\right)\right\} u_{ \pm}\left(r_{l}, \tilde{x}, \lambda\right) \overline{\phi(\lambda, \tilde{x})} d S_{\tilde{x}}
\end{aligned}
$$

So, letting $l \rightarrow \infty$ in $(2.11)$, we have

$$
\begin{aligned}
& -\frac{ \pm}{2 i} \int_{\Omega}\left\{u_{ \pm} \overline{g_{\phi, \pm}}-\overline{f v_{\phi, \pm}}\right\} d x \\
& \quad=\lim _{l \rightarrow \infty}\left(\frac{1}{\sqrt{\pi}} \exp \left\{\rho_{ \pm}\left(r_{l} \cdot, \lambda\right)\right\} u_{ \pm}\left(r_{l} \cdot \lambda\right), \phi(\lambda, \cdot)\right)_{L^{2}\left(S^{n-1}\right)} .
\end{aligned}
$$

By means of (2.10) and Proposition 1.1 (c), the left side of (2.12) equals $\left(f, \frac{ \pm 1}{2 i}\left\{v_{\phi, \pm}-R_{\mp}(\lambda) g_{\phi, \pm}\right\}\right)$. On the other hand, by means of Proposition 1.2 (c) and (1.30), the right side equals $\left(f, \mathscr{F}_{ \pm}^{*}(\lambda) \phi(\lambda, \cdot)\right)$. Thus, we obtain (2.9).

Now we define the operator $K_{ \pm}(e)$ as follows:

$$
\begin{aligned}
& {\left[K_{ \pm}(e) \phi(\cdot, \cdot)\right](x)=\frac{ \pm 1}{2 i} \int_{e} v_{\phi, \pm}(x, \lambda) d \lambda} \\
& \quad= \begin{cases}\frac{ \pm 1}{2 \sqrt{\pi} i} \int_{e} \exp \left\{-\rho_{ \pm}(x, \lambda)\right\} \psi(r) \phi(\lambda, \tilde{x}) d \lambda, & |x|>R_{2}+1 \\
0, & |x| \leq R_{2}+1\end{cases}
\end{aligned}
$$


for $\phi(\lambda, \tilde{x}) \in C_{0}^{\infty}\left(e \times S^{n-1}\right)$. In the next section we shall show that $K_{ \pm}(e)$ can be extended to a bounded operator from $L^{2}\left(e \times S^{n-1}\right)$ to $L^{2}(\Omega)$. The extended operator will be denoted by $K_{ \pm}(e)$ again. Then our identification operators $J_{ \pm}(e)$ are:

$$
J_{ \pm}(e) f=K_{ \pm}(e) \mathscr{F}_{0} f \quad \text { for } \quad f \in \mathscr{E}_{0}(e) L^{2}\left(\boldsymbol{R}^{n}\right)
$$

\section{§3. $\boldsymbol{L}^{2}$-Boundedness of $\boldsymbol{K}_{ \pm}(\boldsymbol{e})$}

We begin with a lemma which is a slight modification of CalderónVaillancourt [6].

Lemma 3.1. Let $I$ be a bounded interval of $\boldsymbol{R}=(-\infty, \infty)$ and let $A(r)$ $(r \in I)$ be a weakly measurable and uniformly bounded family of operators in a separable Hilbert space $\mathfrak{H}$. If the inequalities

$$
\left\|A(r) A^{*}\left(r^{\prime}\right)\right\| \leq h^{2}\left(r, r^{\prime}\right) \quad \text { and } \quad\left\|A^{*}(r) A\left(r^{\prime}\right)\right\| \leq h^{2}\left(r, r^{\prime}\right)
$$

hold for $r, r^{\prime} \in I$ with a non-negative function $h\left(r, r^{\prime}\right)$ which is the kernel of a bounded integral operator $H_{I}$ in $L^{2}(I)\left(A^{*}(r)\right.$ being the adjoint of $\left.A(r)\right)$, then the operator $\int_{I} A(r) d r$ defined by

$$
\left(\int_{I} A(r) d r\right) f=\int_{I} A(r) f d r \quad \text { for } f \in \mathfrak{H}
$$

is a bounded operator in $\mathfrak{H}$ with norm

$$
\left\|\int_{I} A(r) d r\right\| \leq\left\|H_{I}\right\|
$$

Proof. By assumption we can admit $\|A(r)\| \leq M$ for any $r \in I$. From the two inequalities

$$
\left\|A\left(r_{1}\right) A^{*}\left(r_{2}\right) A\left(r_{3}\right) \cdots A^{*}\left(r_{2 m}\right)\right\| \leq\left\|A\left(r_{1}\right) A^{*}\left(r_{2}\right)\right\| \cdots\left\|A\left(r_{2 m-1}\right) A^{*}\left(r_{2 m}\right)\right\|
$$

and

$$
\begin{aligned}
\left\|A\left(r_{1}\right) A^{*}\left(r_{2}\right) A\left(r_{3}\right) \cdots A^{*}\left(r_{2 m}\right)\right\| \leq & \left\|A\left(r_{1}\right)\right\|\left\|A^{*}\left(r_{2}\right) A\left(r_{3}\right)\right\| \cdots \\
& \cdots\left\|A^{*}\left(r_{2 m-2}\right) A\left(r_{2 m-1}\right)\right\|\left\|A^{*}\left(r_{2 m}\right)\right\|,
\end{aligned}
$$

we have for $r_{i} \in I \quad(i=1,2, \cdots, 2 m)$,

(3.1) $\left\|A\left(r_{1}\right) A^{*}\left(r_{2}\right) A\left(r_{3}\right) \cdots A^{*}\left(r_{2 m}\right)\right\| \leq M h\left(r_{1}, r_{2}\right) h\left(r_{2}, r_{3}\right) \cdots h\left(r_{2 m-1}, r_{2 m}\right)$.

Since $\int_{I} A(r) d r\left(\int_{I} A(r) d r\right)^{*}$ is a bounded selfadjoint operator in $\mathfrak{H}$ and 
$\left(\int_{I} A(r) d r\right)^{*}=\int_{I} A^{*}(r) d r$, we have from $(3.1)$

$$
\begin{aligned}
& \left\|\int_{I} A(r) d r\right\|^{2}=\left\|\left[\int_{I} A(r) d r\left(\int_{I} A(r) d r\right)^{*}\right]^{m}\right\|^{1 / m} \\
& \quad \leq\left(\int \cdots \int_{I^{2 m}}\left\|A\left(r_{1}\right) A^{*}\left(r_{2}\right) A\left(r_{3}\right) \cdots A^{*}\left(r_{2 m}\right)\right\| d r_{1} \cdots d r_{2 m}\right)^{1 / m} \\
& \quad \leq\left(M \iint_{I^{2}} d r_{1} d r_{2 m} \int \cdots \int_{I^{2 m-2}} h\left(r_{1}, r_{2}\right) \cdots h\left(r_{2 m-1}, r_{2 m}\right) d r_{2} \cdots d r_{2 m-1}\right)^{1 / m} \\
& \quad \leq\left\{M\left(H_{I}^{2 m-1} \chi_{I}, \chi_{I}\right)_{L^{2}(I)}\right\}^{1 / m} \leq\left(M|I|\left\|H_{I}\right\|^{2 m-1}\right)^{1 / m}
\end{aligned}
$$

where $\chi_{I}(r)=1$ on $I$ and $|I|$ is the length of $I$. Letting $m$ go to $\infty$ in (3.2), we have the assertion.

q.e.d.

Remark 3.1. By Petti's theorem the weak measurability of $A(r)$ and the separability of $\mathfrak{H}$ show that $A(r) f, f \in \mathfrak{H}$, is strongly measurable on $I$. Moreover, $A(r) f$ is Bochner integrable on $I$ since $\|A(r)\|$ is bounded in $I$ (see Yosida [16], pp. 130-134).

Let $e_{0}=\left(\Lambda_{1 / 2}+\varepsilon, \Lambda_{1 / 2}+N\right)$, where $N$ is chosen so large that $e=\left(\lambda_{1}, \lambda_{2}\right)$ $\subset e_{0}$. Let $\zeta(\lambda) \in C_{0}^{\infty}\left(e_{0}\right)$ be a real function such that $\zeta(\lambda)=1$ on $e$, and let $\chi(r) \in$ $C^{\infty}(\boldsymbol{R})$ satisfy the following: $\chi(r)=1$ for $r<1,=0$ for $r>2$ and $0<\chi(r)<1$ for $1<r<2$. We put for $\mu, \lambda \in e_{0}, r>R_{2}+1$ and $\tilde{x} \in S^{n-1}$,

$$
\begin{gathered}
S_{ \pm}(\mu, \lambda, r, \tilde{x})= \pm \int_{R_{2}}^{r}\left\{\sqrt{\lambda-\eta(\lambda) V_{1}(s \tilde{x})}-\sqrt{\mu-\eta(\mu) V_{1}(s \tilde{x})}\right\} d s \\
p_{R}(\mu, \lambda, r, \tilde{x})=\frac{1}{4 \pi} \psi(r)^{2} \chi(r / R)^{2} \zeta(\lambda) \zeta(\mu) \\
\quad \times\left\{\lambda-\eta(\lambda) V_{1}(r \tilde{x})\right\}^{-1 / 4}\left\{\mu-\eta(\mu) V_{1}(r \tilde{x})\right\}^{-1 / 4}
\end{gathered}
$$

where $\psi(r)$ is as given in (2.1) and $R \geq\left(R_{2}+1\right) / 2$.

For any $r, r^{\prime}>R_{2}+1$, we have

$$
\begin{array}{r}
S_{ \pm}(\mu, \xi, r, \tilde{x})+S_{ \pm}\left(\xi, \lambda, r^{\prime}, \tilde{x}\right)= \pm \int_{r^{\prime}}^{r} \sqrt{\xi-\eta(\xi) V_{1}(s \tilde{x})} d s \\
\quad \pm\left\{\int_{R_{2}}^{r^{\prime}} \sqrt{\lambda-\eta(\lambda) V_{1}(s \tilde{x})} d s-\int_{R_{2}}^{r} \sqrt{\mu-\eta(\mu) V_{1}(s \tilde{x})} d s\right\}
\end{array}
$$

and

$$
\begin{aligned}
& \partial_{\xi}^{3} \exp \left\{ \pm i \int_{r^{\prime}}^{r} \sqrt{\xi-\eta(\xi) V_{1}(s \tilde{x})} d s\right\} \\
& \quad=\sigma_{ \pm}\left(\xi, r, r^{\prime}, \tilde{x}\right) \exp \left\{ \pm i \int_{r^{\prime}}^{r} \sqrt{\xi-\eta(\xi) V_{1}(s \tilde{x})} d s\right\}
\end{aligned}
$$




$$
\begin{aligned}
& \sigma_{ \pm}\left(\xi, r, r^{\prime}, \tilde{x}\right) \\
& =\mp i\left(\int_{r^{\prime}}^{r} \partial_{\xi} \sqrt{\xi-\eta(\xi) V_{1}(s \tilde{x})} d s\right)^{3} \pm i \int_{r^{\prime}}^{r} \partial_{\xi}^{3} \sqrt{\xi-\eta(\xi) V_{1}(s \tilde{x})} d s \\
& \quad-3 \int_{r^{\prime}}^{r} \partial_{\xi} \sqrt{\xi-\eta(\xi) V_{1}(s \tilde{x})} d s\left(\int_{r^{\prime}}^{r} \partial_{\xi}^{2} \sqrt{\xi-\eta(\xi) V_{1}(s \tilde{x})} d s\right) .
\end{aligned}
$$

Then the following inequalities are consequences of Lemma 1.2. Namely, there exists a constant $C_{8} \geq 1$ such that for any $r, r^{\prime} \geq R_{2}+1, \xi, \mu, \lambda \in e_{0}, \tilde{x} \in S^{n-1}$ and $R \geq\left(R_{2}+1\right) / 2$,

$$
\begin{aligned}
& \left|\sigma_{ \pm}\left(\xi, r, r^{\prime}, \tilde{x}\right)\right| \geq C_{8}^{-1}\left|r-r^{\prime}\right|^{3}-C_{8}\left|r-r^{\prime}\right|, \\
& \left|\partial_{\xi}^{l} \sigma_{ \pm}\left(\xi, r, r^{\prime}, \tilde{x}\right)\right| \leq C_{8}\left(1+\left|r-r^{\prime}\right|^{3}\right) \quad(l=1,2,3), \\
& \left|\partial_{\xi}^{l}\left[p_{R}(\mu, \xi, r, \tilde{x}) p_{R}\left(\xi, \lambda, r^{\prime}, \tilde{x}\right)\right]\right| \leq C_{8} \quad(l=0,1,2,3) .
\end{aligned}
$$

With these inequalities we can apply Lemma 3.1 to prove the following

Lemma 3.2. The operator $P_{R, \pm}$ defined by

$$
\left[P_{R, \pm} \phi\right](\mu, \tilde{x})=\int_{R_{2}+1}^{\infty} \int_{e_{0}} \exp \left\{i S_{ \pm}(\mu, \lambda, r, \tilde{x})\right\} p_{R}(\mu, \lambda, r, \tilde{x}) \phi(\lambda, \tilde{x}) d r d \lambda
$$

for $\phi(\lambda, \tilde{x}) \in \mathfrak{H}=L^{2}\left(e_{0} \times S^{n-1}\right)$ is bounded in $\mathfrak{H}$, and there exists a constant $C_{9}>0$ such that

$$
\left\|P_{R, \pm}\right\| \leq C_{9} \text { for any } R \geq\left(R_{2}+1\right) / 2 \text {. }
$$

Proof. We define the family $A_{R, \pm}(r), r \in I_{R}=\left(R_{2}+1,2 R\right)$, of operators in $\mathfrak{S}$ by

$$
\left[A_{R, I}(r) \phi\right](\mu, \tilde{x})=\int_{e_{0}} \exp \left\{i S_{ \pm}(\mu, \lambda, r, \tilde{x})\right\} p_{R}(\mu, \lambda, r, \tilde{x}) \phi(\lambda, \tilde{x}) d \lambda .
$$

Obviously, each $A_{R, \pm}(r)$ is bounded and selfadjoint in $\mathfrak{H}$. Since we have

$$
\left\|A_{R, \pm}(r)\right\| \leq\left\{\sup _{\tilde{x} \in S^{n-1}} \int_{e_{0}} \int_{e_{0}}\left|p_{R}(\mu, \lambda, r, \tilde{x})\right|^{2} d \lambda d \mu\right\}^{1 / 2}
$$

it follows from (3.10) that

$$
\left\|A_{R, \pm}(r)\right\| \leq C_{10} \quad \text { for any } \quad R \geq\left(R_{2}+1\right) / 2 \quad \text { and } \quad r \in I_{R} .
$$

Further, by the Lebesgue theorem, $A_{R, \pm}(r)$ is strongly continuous in $I_{R}$. Thus, to complete the proof we have only to show the existence of a kernel $h_{R}\left(r, r^{\prime}\right)$ which satisfies the following inequalities:

$$
\begin{aligned}
& \left\|A_{R, \pm}(r) A_{R, \pm}\left(r^{\prime}\right)\right\| \leq h_{R}^{2}\left(r, r^{\prime}\right), \\
& \int_{I_{R}}\left|\int_{I_{R}} h_{R}\left(r, r^{\prime}\right) f\left(r^{\prime}\right) d r^{\prime}\right|^{2} d r \leq C_{11} \int_{I_{R}}\left|f\left(r^{\prime}\right)\right|^{2} d r^{\prime}
\end{aligned}
$$


for any $R \geq\left(R_{2}+1\right) / 2, r, r^{\prime} \in I_{R}$ and $f(r) \in L^{2}\left(I_{R}\right)$, where $C_{11}>0$ is independent of $R$.

We can choose $C_{12}>0$ and $C_{13}>0$ to satisfy

$$
C_{12}+C_{8}^{-1} \tau^{3}-C_{8} \tau \geq C_{13}\left(1+\tau^{3}\right) \quad \text { for any } \tau \geq 0 .
$$

It then follows from (3.8) that

$$
\left|\mp i C_{12} \operatorname{sgn}\left(r-r^{\prime}\right)+\sigma_{ \pm}\left(\xi, r, r^{\prime}, \tilde{x}\right)\right| \geq C_{13}\left(1+\left|r-r^{\prime}\right|^{3}\right),
$$

where $\operatorname{sgn} t=1$ if $t \geq 0$ and $=-1$ if $t<0$. So by (3.6),

$$
\begin{aligned}
& \exp \left\{ \pm i \int_{r^{\prime}}^{r} \sqrt{\xi-\eta(\xi) V_{1}(s \tilde{x})} d s\right\}=\left\{\mp i C_{12} \operatorname{sgn}\left(r-r^{\prime}\right)+\sigma_{ \pm}\left(\xi, r, r^{\prime}, \tilde{x}\right)\right\}^{-1} \\
& \quad \times\left\{\mp i C_{12} \operatorname{sgn}\left(r-r^{\prime}\right)+\partial_{\xi}^{3}\right\} \exp \left\{ \pm i \int_{r^{\prime}}^{r} \sqrt{\xi-\eta(\xi) V_{1}(s \tilde{x})} d s\right\}
\end{aligned}
$$

Note that the support in $\xi$ of $p_{R}(\mu, \xi, r, \tilde{x}) p_{R}\left(\xi, \lambda, r^{\prime}, \tilde{x}\right)$ is contained in $e_{0}$. Then (3.5), (3.19) and integrations by parts give

$$
\begin{aligned}
\int_{e_{0}} & \exp \left\{i S_{ \pm}(\mu, \xi, r, \tilde{x})+i S_{ \pm}\left(\xi, \lambda, r^{\prime}, \tilde{x}\right)\right\} p_{R}(\mu, \xi, r, \tilde{x}) p_{R}\left(\xi, \lambda, r^{\prime}, \tilde{x}\right) d \xi \\
= & \int_{e_{0}} \exp \left\{i S_{ \pm}(\mu, \xi, r, \tilde{x})+i S_{ \pm}\left(\xi, \lambda, r^{\prime}, \tilde{x}\right)\right\}\left\{\mp i C_{12} \operatorname{sgn}\left(r-r^{\prime}\right)-\partial_{\xi}^{3}\right\} \\
& \times\left[\left\{\mp i C_{12} \operatorname{sgn}\left(r-r^{\prime}\right)+\sigma_{ \pm}\left(\xi, r, r^{\prime}, \tilde{x}\right)\right\}^{-1} p_{R}(\mu, \xi, r, \tilde{x}) p_{R}\left(\xi, \lambda, r^{\prime}, \tilde{x}\right)\right] d \xi
\end{aligned}
$$

Applying (3.9), (3.10) and (3.18) in this equality, we obtain

$$
\begin{aligned}
& \left|\int_{e_{0}} \exp \left\{i S_{ \pm}(\mu, \xi, r, \tilde{x})+i S_{ \pm}\left(\xi, \lambda, r^{\prime}, \tilde{x}\right)\right\} p_{R}(\mu, \xi, r, \tilde{x}) p_{R}\left(\xi, \lambda, r^{\prime}, \tilde{x}\right) d \xi\right| \\
& \quad \leq C_{14}\left(1+\left|r-r^{\prime}\right|^{3}\right)^{-1}
\end{aligned}
$$

where $C_{14}>0$ is independent of $R \geq\left(R_{2}+1\right) / 2, r, r^{\prime} \in I_{R}, \mu, \lambda \in e_{0}$ and $\tilde{x} \in S^{n-1}$.

Now for any $\phi(\lambda, \tilde{x}) \in \mathfrak{H}$,

$$
\begin{aligned}
& {\left[A_{R, \pm}(r) A_{R, \pm}\left(r^{\prime}\right) \phi\right](\mu, \tilde{x})} \\
& \quad=\int_{e_{0}} \phi(\lambda, \tilde{x}) d \lambda \int_{e_{0}} \exp \left\{i S_{ \pm}(\mu, \xi, r, \tilde{x})+i S_{ \pm}\left(\xi, \lambda, r^{\prime}, \tilde{x}\right)\right\} \\
& \quad \times p_{R}(\mu, \xi, r, \tilde{x}) p_{R}\left(\xi, \lambda, r^{\prime}, \tilde{x}\right) d \xi .
\end{aligned}
$$

So (3.20) has shown the inequality

$$
\left\|A_{R, \pm}(r) A_{R, \pm}\left(r^{\prime}\right)\right\| \leq C_{14}^{\prime}\left(1+\left|r-r^{\prime}\right|^{3}\right)^{-1} \quad \text { with } \quad C_{14}^{\prime}=C_{14}\left|e_{0}\right| .
$$

Hence, choosing $h_{R}\left(r, r^{\prime}\right)=\sqrt{C_{14}^{\prime}}\left(1+\left|r-r^{\prime}\right|^{3}\right)^{-1 / 2}$ for any $R \geq\left(R_{2}+1\right) / 2$, we have (3.16) and (3.17) with $C_{11}=C_{14}^{\prime}\left\{\int_{\mathbb{R}}\left(1+r^{3}\right)^{-1 / 2} d r\right\}^{2}<\infty$.

q.e.d.

Remark 3.2. The method of the above proof, which apparently seems to 
be much different, however, follows the idea employed in Calderón-Vaillancourt [6]. If $V_{1}(x)$ is sufficiently smooth, e.g., $\in C^{19}\left(B\left(R_{2}\right)\right)$, a general theory of Asada-Fujiwara [3] can be applied to obtain the above result.

As a corollary of Lemma 3.2 we can now prove the following

Proposition 3.1. For any $\phi(\lambda, \tilde{x}) \in C_{0}^{\infty}\left(e \times S^{n-1}\right)$, where $e \Subset e_{0}$, let $K_{ \pm}(e) \phi$ be defined by (2.13). Then we have $K_{ \pm}(e) \phi \in L^{2}(\Omega)$ and

$$
\left\|K_{ \pm}(e) \phi\right\|^{2} \leq C_{9}\|\phi\|_{L^{2}\left(e \times S^{n-1}\right)}^{2} .
$$

Thus, $K_{ \pm}(e)$ can be extended to a bounded operator from $L^{2}\left(e \times S^{n-1}\right)$ to $L^{2}(\Omega)$.

Proof. By integration by parts we have

$$
\begin{aligned}
{\left[K_{ \pm}\right.} & (e) \phi](x)=\frac{ \pm 1}{2 \sqrt{\pi i}} r^{-(n-1) / 2} \psi(r) \\
& \times \int_{e} \exp \left\{ \pm i \int_{R_{2}}^{r} \sqrt{\lambda-\eta(\lambda) V_{1}(s \tilde{x})} d s\right\}\left\{\lambda-\eta(\lambda) V_{1}(x)\right\}^{-1 / 4} \phi(\lambda, \tilde{x}) d \lambda \\
= & \frac{1}{2 \sqrt{\pi}} r^{-(n-1) / 2} \psi(r) \int_{e} \exp \left\{ \pm i \int_{R_{2}}^{r} \sqrt{\lambda-\eta(\lambda) V_{1}(s \tilde{x})} d s\right. \\
& \times \partial_{\lambda}\left[\left\{\int_{R_{2}}^{r} \partial_{\lambda} \sqrt{\lambda-\eta(\lambda) V_{1}(s \tilde{x})} d s\right\}^{-1}\left\{\lambda-\eta(\lambda) V_{1}(x)\right\}^{-1 / 4} \phi(\lambda, \tilde{x})\right] d \lambda .
\end{aligned}
$$

This with Lemma 1.2 shows that

$$
\left|\left[K_{ \pm}(e) \phi\right](x)\right| \leq C_{15} r^{-(n-1) / 2} r^{-1} \text { in } B\left(R_{2}+1\right),
$$

i.e., $K_{ \pm}(e) \phi \in L^{2}(\Omega)$. Thus, we can apply the Lebesgue theorem and the Fubini theorem to obtain

$$
\begin{aligned}
& \left\|K_{ \pm}(e) \phi\right\|^{2}=\lim _{R \rightarrow \infty} \int_{\Omega}\left|\chi(r / R)\left[K_{ \pm}(e) \phi\right](x)\right|^{2} d x \\
& =\lim _{R \rightarrow \infty} \int_{e} \int_{S^{n-1}} \overline{\phi(\mu, \tilde{x})} d \mu d S \int_{R_{2}}^{\infty} \int_{e} \exp \left\{i S_{ \pm}(\mu, \lambda, r, \tilde{x})\right\} \\
& \quad \times p_{R}(\mu, \lambda, r, \tilde{x}) \phi(\lambda, \tilde{x}) d r d \lambda=\lim _{R \rightarrow \infty}\left(P_{R, \pm} \phi, \phi\right)_{L^{2}\left(e \times S^{n-1}\right)} \\
& =\lim _{R \rightarrow \infty}\left(P_{R, \pm} \phi, \phi\right)_{L^{2}\left(e_{0} \times S^{n-1}\right)} .
\end{aligned}
$$

(3.12) and (3.24) imply (3.22).

q.e.d.

\section{§4. Theorem; Time Dependent Representations of $\mathbb{U}_{ \pm}(e)$}

First we note the following lemma which can easily be proved by Lemma 1.2. 
Lemma 4.1. Let $\varepsilon$ and $N$ be any constants satisfying $0<\varepsilon<1$ and $N>\Lambda_{\tilde{\delta}}-\Lambda_{1 / 2}+2$. Then there exist some constants $C_{16} \geq 1$ and $R_{2} \geq R_{1}^{\prime}$ such that for any $(x, \lambda) \in B\left(R_{2}\right) \times\left[\Lambda_{\tilde{\delta}}+2 \varepsilon, \Lambda_{1 / 2}+N\right]$,

$$
C_{16}^{-1} \leq-\partial_{\lambda}^{2} \sqrt{\lambda-\eta(\lambda) V_{1}(x)} \leq C_{16} .
$$

In this and next section we choose $R_{2} \geq R_{1}^{\prime}$ defining $\rho_{ \pm}(\lambda, \lambda)$ as in the above lemma, and prove the following theorem which gives time dependent representations of the stationary wave operators $U_{ \pm}(e)$ with $e \subset\left[\Lambda_{\tilde{\delta}}+2 \varepsilon, \Lambda_{1 / 2}+N\right]$. Note that the operators $U_{ \pm}(e)$ and $J_{ \pm}(e)$ and functions $v_{\phi, \pm}(x, \lambda)$ and $g_{\phi, \pm}(x, \lambda)$ are now determined depending on the above $R_{2}$.

Theorem. Let $\varepsilon, N$ and $R_{2}$ be as in the above lemma. For any interval $e=\left(\lambda_{1}, \lambda_{2}\right) \subset\left[\Lambda_{\tilde{\delta}}+2 \varepsilon, \Lambda_{1 / 2}+N\right]$ let $J_{ \pm}(e): \mathscr{E}_{0}(e) L^{2}\left(\mathbb{R}^{n}\right) \rightarrow L^{2}(\Omega)$ be defined by (2.14). Then the strong limits

$$
W_{J}^{ \pm}(e)=s-\lim _{t \rightarrow \pm \infty} \exp \{i L t\} J_{ \pm}(e) \exp \left\{-i \mathbb{L}_{0} \hat{t}\right\} \mathscr{E}_{0}(e)
$$

exist in $L^{2}(\Omega)$ and coincide with the stalionary wave operators $U_{ \pm}(e)$ defined by (1.33). Thus, $W_{\bar{J}}^{ \pm}(e)$ are unitary operators from $\mathscr{E}_{0}(e) L^{2}\left(\mathbb{R}^{n}\right)$ onto $\mathscr{E}(e) L^{2}(\Omega)$ satisfying

$$
\mathscr{E}(e) L W_{J}^{ \pm}(e) f=W_{J}^{ \pm}(e) \mathscr{E}_{0}(e) L_{0} f \text { for any } f \in \mathscr{D}\left(L_{0}\right) .
$$

Remark 4.1. $K_{ \pm}(e)$ and $J_{ \pm}(e)$ depend on the function $\psi(r)$ given in (2.1). However, $W_{J}^{ \pm}(e)$ does not depend on the choice of $\psi(r)$.

The following proposition will be proved in the next section by use of Lemma 4.1 and the stationary phase method.

Proposition 4.1. For $\phi(\lambda, \tilde{x}) \in C_{0}^{\infty}\left(e \times S^{n-1}\right)$ let

$$
\hat{g}_{\phi, \pm}(x, t)=\int_{e} \exp \{-i \lambda t\} g_{\phi, \pm}(x, \lambda) d \lambda \quad( \pm t>0),
$$

where $g_{\phi, \pm}(x, \lambda)$ is defined by (2.2). Then we have

$$
\pm \int_{0}^{ \pm \infty}\left\|\hat{g}_{\phi, \pm}(\cdot, t)\right\| d t<\infty
$$

Based on Propositions 2.1, 3.1 and 4.1, we can now follow the idea employed in Kitada [13], Ikebe-Isozaki [10] and Kako [11], where is treated the case of "non-oscillating"' long-range potentials, to prove the above theorem.

Lemma 4.2. We have for any $\phi(\lambda, \tilde{x}) \in C_{0}^{\infty}\left(e \times S^{n-1}\right)$, 


$$
\left\|\int_{e} R_{\mp}(\lambda) g_{\phi, \pm}(\cdot, \lambda) d \lambda\right\| \leq \pm \int_{0}^{ \pm \infty}\left\|\hat{g}_{\phi, \pm}(\cdot, t)\right\| d t
$$

Proof. Noting that $g_{\phi, \pm}(\cdot, \lambda) \in L_{(1+\tilde{\beta}) / 2}^{2}(\Omega)$, where $\tilde{\beta}$ is as given in Proposition 1.3 , we put

$$
\begin{aligned}
& G_{ \pm}(x)=\int_{e} R_{\mp}(\lambda) g_{\phi, \pm}(x, \lambda) d \lambda, \\
& G_{\tau, \pm}(x)=\int_{e} R(\lambda \mp i \tau) g_{\phi, \pm}(x, \lambda) d \lambda \quad(\tau>0),
\end{aligned}
$$

where the measurability of the integrands is guaranteed by Proposition 1.1 (b) and the continuity of $g_{\phi, \pm}(\cdot, \lambda)$ in $\lambda \in e$. In virtue of $(2.4)$ we have

$$
\begin{aligned}
G_{\tau, \pm}(x) & =-i \int_{e}\left[\int_{0}^{ \pm \infty} \exp \{i(L-\lambda \pm i \tau) t\} d t\right] g_{\phi, \pm}(x, \lambda) d \lambda \\
& =-i \int_{0}^{ \pm \infty} \exp \{i(L \pm i \tau) t\} \hat{g}_{\phi, \pm}(x, t) d t .
\end{aligned}
$$

Thus,

$$
\left\|G_{\tau, \pm}\right\| \leq \pm \int_{0}^{ \pm \infty}\left\|\hat{g}_{\phi, \pm}(\cdot, t)\right\| d t<\infty \quad \text { for any } \quad \tau>0 .
$$

Further, since we have for any $f \in L_{(1+\tilde{\beta}) / 2}^{2}(\Omega)$ and $\tau>0$,

$$
\left(G_{\tau, \pm}, f\right)=\int_{e}\left(g_{\phi, \pm}(\cdot, \lambda), R(\lambda \pm i \tau) f\right) d \lambda,
$$

it follows from Proposition 1.1 (a), (b), (c) and the Lebesgue theorem that

$$
\begin{aligned}
\lim _{\tau \downarrow 0}\left(G_{\tau, \pm}, f\right) & =\int_{e}\left(g_{\phi, \pm}(\cdot, \lambda), R_{ \pm}(\lambda) f\right) d \lambda \\
& =\int_{e}\left(R_{\mp}(\lambda) g_{\phi, \pm}(\cdot, \lambda), f\right) d \lambda=\left(G_{ \pm}, f\right) .
\end{aligned}
$$

$L_{(1+\tilde{\beta}) / 2}^{2}(\Omega)$ being dense in $L^{2}(\Omega)$, this and (4.7) imply that $G_{ \pm}$is the weak limit as $\tau \downarrow 0$ of $G_{\tau, \pm}$ in $L^{2}(\Omega)$. Hence, $G_{ \pm} \in L^{2}(\Omega)$ and

$$
\left\|G_{ \pm}\right\| \leq \liminf _{\tau \downarrow 0}\left\|G_{\tau, \pm}\right\| \leq \pm \int_{0}^{ \pm \infty}\left\|\hat{g}_{\phi, \pm}(\cdot, t)\right\| d t,
$$

which is to be proved.

q.e.d.

Proof of Theorem. Let $f \in \mathscr{E}_{0}(e) L^{2}\left(\boldsymbol{R}^{n}\right)$ satisfy $\left[\mathscr{F}_{0} f\right](\lambda, \tilde{x}) \in C_{0}^{\infty}\left(e \times S^{n-1}\right)$, and put $u(t)=\exp \left\{-i L_{0} t\right\} f$. Since $\mathscr{F}_{0} u(t)=\exp \{-i \lambda t\} \mathscr{F}_{0} f$ by Proposition 1.3 (c), we see that $\mathscr{F}_{0} u(t)$ also belongs to $C_{0}^{\infty}\left(e \times S^{n-1}\right)$. By Propositions 1.3 (d) and 2.1 we then have 


$$
\begin{aligned}
& \exp \{-i L t\} U_{ \pm}(e) f=U_{ \pm}(e) u(t) \\
& \quad=\frac{ \pm 1}{2 i} \int_{e}\left\{v_{\mathscr{F}_{0} u(t), \pm}(\cdot, \lambda)-R_{\mp}(\lambda) g_{\mathscr{F}_{0} u(t), \pm}(\cdot, \lambda)\right\} d \lambda
\end{aligned}
$$

Here by definition

$$
\frac{ \pm 1}{2 i} \int_{e} v_{\mathscr{F}_{0} u(t), \pm}(\cdot, \lambda) d \lambda=J_{ \pm}(e) u(t)=J_{ \pm}(e) \exp \left\{-i L_{0} t\right\} f
$$

On the other hand, the equality

$$
g_{\mathscr{F}_{0} u(t), \pm}(\cdot, \lambda)=\exp \{-i \lambda t\} g_{\mathscr{F}_{0} f, \pm}(\cdot, \lambda)
$$

and (4.4) show that

$$
\hat{g}_{\mathscr{F}_{0} u(t), \pm}(\cdot, s)=\hat{g}_{\mathscr{F}_{0} f, \pm}(\cdot, s+t) \quad \text { for any } \pm s>0,
$$

and hence, we have from Lemma 4.2 and Proposition 4.1,

$$
\begin{gathered}
\left\|\int_{e} R_{\mp}(\lambda) g_{\mathscr{F}_{0} u(t), \pm}(\cdot, \lambda) d \lambda\right\| \leq \pm \int_{0}^{ \pm \infty}\left\|\hat{g}_{\mathscr{F}_{0} f, \pm}(\cdot, s+t)\right\| d s \\
= \pm \int_{t}^{ \pm \infty}\left\|\hat{g}_{\mathscr{F}_{0} f, \pm}(\cdot, s)\right\| d s \longrightarrow 0 \text { as } t \longrightarrow \pm \infty
\end{gathered}
$$

(4.8), (4.9) and (4.10) prove the following:

$$
\lim _{t \rightarrow \pm \infty}\left\|\exp \{i L t\} J_{ \pm}(e) \exp \left\{-i L_{0} t\right\} f-U_{ \pm}(e) f\right\|=0 .
$$

Since $C_{0}^{\infty}\left(e \times S^{n-1}\right)$ is dense in $L^{2}\left(e \times S^{n-1}\right)$ and $\mathscr{F}_{0}$ is a unitary operator from $\mathscr{E}_{0}(e) L^{2}\left(\mathbb{R}^{n}\right)$ onto $L^{2}\left(e \times S^{n-1}\right),(4.11)$ holds for any $f \in \mathscr{E}_{0}(e) L^{2}\left(\mathbb{R}^{n}\right)$.

The proof is thus completed.

q.e.d.

\section{§5. Proof of Proposition 4.1; The Stationary Phase Method}

We put for the sake of simplicity

$$
\begin{aligned}
& \xi(x, \lambda)=\int_{R_{2}}^{r} \sqrt{\lambda-\eta(\lambda) V_{1}(s \tilde{x})} d s, \\
& \zeta_{\phi, \pm}(x, \lambda)=\sqrt{\pi} \exp \left\{\rho_{ \pm}(x, \lambda)\right\} g_{\phi, \pm}(x, \lambda),
\end{aligned}
$$

where $\phi(\lambda, \tilde{x}) \in C_{0}^{\infty}\left(e \times S^{n-1}\right)$ with $e=\left(\lambda_{1}, \lambda_{2}\right) \subset\left[\Lambda_{\tilde{\delta}}+2 \varepsilon, \Lambda_{1 / 2}+N\right]$. We can find a concrete form of $\zeta_{\phi, \pm}(x, \lambda)$ in (2.3).

The following lemma is easily proved by a straight calculation (cf. Lemmas 1.2, 1.3 and 4.1).

Lemma 5.1. There exists a constant $C_{17} \geq 1$ such that for any $(x, \lambda)$ $\in B\left(R_{2}+1\right) \times e$, 


$$
\begin{aligned}
& C_{17}^{-1} r \leq \partial_{\lambda} \xi(x, \lambda) \leq C_{17} r, \\
& C_{17}^{-1} r \leq-\partial_{\lambda}^{2} \xi(x, \lambda) \leq C_{17} r, \\
& \left|\partial_{\lambda}^{l} \xi(x, \lambda)\right| \leq C_{17} r \quad(l=3,4,5), \\
& \left|\partial_{\lambda}^{l} \zeta_{\phi, \pm}(x, \lambda)\right| \leq C_{17} r^{-1-\tilde{\delta}} \quad(l=0,1,2) .
\end{aligned}
$$

Let $e_{1}=\left[\lambda_{3}, \lambda_{4}\right] \subset e$ be a closed interval which contains the support in $\lambda$ of $\zeta_{\phi, \pm}(x, \lambda)$ for any $x \in B\left(R_{2}+1\right)$. We put

$$
t_{j}(x)=\left(\partial_{\lambda} \xi\right)\left(x, \lambda_{j}\right)
$$

Then we have

$$
C_{17}^{-1} r \leq t_{2}(x)<t_{4}(x)<t_{3}(x)<t_{1}(x) \leq C_{17} r
$$

since $\left(\partial_{\lambda} \xi\right)(x, \lambda)$ is by $(5.4)$ a monotone decreasing (in a strong sense) function of $\lambda \in e$ for any $x \in B\left(R_{2}+1\right)$. Moreover, we have the

Lemma 5.2. There exists a constant $C_{18} \geq 1$ such that for any $x \in B\left(R_{2}+1\right)$,

$$
\begin{aligned}
& C_{18}^{-1} r \leq t_{1}(x)-t_{3}(x) \leq C_{18} r, \\
& C_{18}^{-1} r \leq t_{4}(x)-t_{2}(x) \leq C_{18} r .
\end{aligned}
$$

Proof. Since we have

$$
t_{1}(x)-t_{3}(x)=\left(\lambda_{1}-\lambda_{3}\right) \partial_{\lambda}^{2} \xi\left(x, \lambda_{1}+\left(\lambda_{3}-\lambda_{1}\right) \theta\right)
$$

for a suitable $\theta(0<\theta=\theta(x)<1),(5.9)$ is a consequence of (5.4). (5.10) can similarly be proved.

With the above lemmas we shall estimate the function

$$
\begin{aligned}
& \hat{g}_{\phi, \pm}(x, t)=\int_{e} \exp \{-i \lambda t\} g_{\phi, \pm}(x, \lambda) d \lambda \\
& \quad=\frac{1}{\sqrt{\pi}} r^{-(n-1) / 2} \int_{e_{1}} \exp \{-i \lambda t \pm i \xi(x, \lambda)\}\left\{\lambda-\eta(\lambda) V_{1}(x)\right\}^{-1 / 4} \zeta_{\phi, \pm}(x, \lambda) d \lambda
\end{aligned}
$$

Our estimation will be done in the each case $\pm t>t_{1}(x), 0 \leq \pm t<t_{2}(x)$ or $t_{2}(x) \leq \pm t \leq t_{1}(x)$.

In the case $\pm t>t_{1}(x)$ or $0 \leq \pm t<t_{2}(x)$, it holds that

$$
\left|\partial_{\lambda}\{\lambda t \mp \xi(x, \lambda)\}\right|=\left|t-\partial_{\lambda} \xi(x, \lambda)\right| \geq|t|-t_{3}(x) \text { or } t_{4}(x)-|t|
$$

for any $(x, \lambda) \in B\left(R_{2}+1\right) \times e_{1}$. So we can prove the

Lemma 5.3. There exists a $C_{19}>0$ such that

$$
\left|\hat{g}_{\phi, \pm}(x, t)\right| \leq C_{19} r^{-(n-1) / 2} r^{-1-\tilde{\delta}}\left\{|t|-t_{3}(x)\right\}^{-2}\left[1+r^{2}\left\{|t|-t_{3}(x)\right\}^{-2}\right]
$$


for any $x \in B\left(R_{2}+1\right)$ and $\pm t>t_{1}(x)$, and

$$
\left|\hat{g}_{\phi, \pm}(x, t)\right| \leq C_{19} r^{-(n-1) / 2} r^{-1-\tilde{\delta}}\left\{t_{4}(x)-|t|\right\}^{-2}\left[1+r^{2}\left\{t_{4}(x)-|t|\right\}^{-2}\right]
$$

for any $x \in B\left(R_{2}+1\right)$ and $0 \leq \pm t<t_{2}(x)$.

Proof. Integrating by parts gives

$$
\begin{aligned}
\hat{g}_{\phi, \pm}(x, t)=\frac{1}{\sqrt{\pi}} r^{-(n-1) / 2} \int_{e_{1}}[ & \left.\left\{\frac{1}{\partial_{\lambda}(-i \lambda t \pm i \xi)} \partial_{\lambda}\right\}^{2} \exp \{-i \lambda t \pm i \xi(x, \lambda)\}\right] \\
& \times\left\{\lambda-\eta(\lambda) V_{1}(x)\right\}^{-1 / 4} \zeta_{\phi, \pm}(x, \lambda) d \lambda \\
= & -\frac{1}{\sqrt{\pi}} r^{-(n-1) / 2} \int_{e_{1}} \exp \{-i \lambda t \pm i \xi(x, \lambda)\}\left(t \mp \partial_{\lambda} \xi\right)^{-2} \\
\times & \times\left[\partial_{\lambda}^{2}\left\{\left(\lambda-\eta V_{1}\right)^{-1 / 4} \zeta_{\phi, \pm}\right\} \pm\right. \\
+ & 3\left(\partial_{\lambda}^{2} \xi\right)\left(t \mp \partial_{\lambda} \xi\right)^{-1} \partial_{\lambda}\left\{\left(\lambda-\eta V_{1}\right)^{-1 / 4} \zeta_{\phi, \pm}\right\} \\
+ & \left.\left\{3\left(\partial_{\lambda}^{2} \xi\right)^{2}\left(t \mp \partial_{\lambda} \xi\right)^{-2} \pm\left(\partial_{\lambda}^{3} \xi\right)\left(1 \mp \partial_{\lambda} \xi\right)^{-1}\right\}\left(\lambda-\eta V_{1}\right)^{-1 / 4 \zeta_{\phi, \pm}}\right] d \lambda .
\end{aligned}
$$

Thus, noting (5.12), (5.4), (5.5) and the inequality

$$
\left|\partial_{\lambda}^{l}\left\{\left(\lambda-\eta V_{1}\right)^{-1 / 4} \zeta_{\phi, \pm}\right\}\right| \leq C_{20} r^{-1-\tilde{\delta}} \quad(l=0,1,2)
$$

which follows from (5.6) and Lemma 1.2, we obtain $(5,13)$ and (5.14). q.e.d.

Next we consider the case $t_{2}(x) \leq \pm t \leq t_{1}(x)$.

Lemma 5.4. There exists a (unique) function $\lambda_{c}(x, t)$ such that for any $x \in B\left(R_{2}+1\right)$ and $t_{2}(x) \leq \pm t \leq t_{1}(x)$,

$$
\begin{aligned}
& |t|=\left(\partial_{\lambda} \dot{\xi}\right)\left(x, \lambda_{c}(x, t)\right), \\
& \lambda_{1} \leq \lambda_{c}(x, t) \leq \lambda_{2}, \\
& \lambda_{c}(x,-t)=\lambda_{c}(x, t) .
\end{aligned}
$$

Proof. We have only to solve in $\lambda$ the equation $|t|=(\partial, \xi)(x, \lambda)$, which is possible by the monotonicity of $\left(\partial_{\lambda} \xi\right)(x, \lambda)$.

q.e.d.

$\lambda_{c}(x, t)$ is the so-called critical point of $\lambda|t|-\xi(x, \lambda)$.

Let $\omega(\lambda)$ be a $C^{\infty}$-function of $\lambda \in \mathbb{R}$ such that $0 \leq \omega(\lambda) \leq 1, \omega(\lambda)=1$ for $|\lambda|$ $\leq 1 / 2$ and $=0$ for $|\lambda| \geq 1$. By use of this function we divide $\hat{g}_{\phi . \pm}(x, t)$ into two parts:

$$
\begin{aligned}
\hat{g}_{\phi, \pm}(x, t)= & \frac{1}{\sqrt{\pi}} r^{-(n-1) / 2} \int_{e_{1}} \exp \{-i \lambda t \pm i \xi(x, \lambda)\} \\
& \times \omega\left(v(x, t)\left\{\lambda-\lambda_{c}(x, t)\right\}\right)\left\{\lambda-\eta V_{1}(x)\right\}^{-1 / 4} \zeta_{\phi, \pm}(x, \lambda) d \lambda \\
& +\frac{1}{\sqrt{\pi}} r^{-(n-1) / 2} \int_{e_{1}} \exp \{-i \lambda t \pm i \xi(x, \lambda)\} \\
& \times\left\{1-\omega\left(v(x, t)\left\{\lambda-\lambda_{c}(x, t)\right\}\right)\right\}\left\{\lambda-\eta V_{1}(x)\right\}^{-1 / 4} \zeta_{\phi, \pm}(x, \lambda) d \lambda \\
= & \hat{g}_{\phi, \pm}^{(1)}(x, t)+\hat{g}_{\phi, \pm}^{(2)}(x, t),
\end{aligned}
$$


where $v(x, t) \geq 1$ is given later. Note that

$$
\begin{array}{r}
\lambda t \mp \xi(x, \lambda)=\lambda_{c} t \mp \xi\left(x, \lambda_{c}\right) \mp \frac{1}{2}\left(\lambda-\lambda_{c}\right)^{2}\left(\partial_{\lambda}^{2} \xi\right)\left(x, \lambda_{c}\right) \\
\mp \frac{1}{2}\left(\lambda-\lambda_{c}\right)^{3} \int_{0}^{1}(1-\tau)^{2}\left(\partial_{\lambda}^{3} \xi\right)\left(x, \lambda_{c}+\left(\lambda-\lambda_{c}\right) \tau\right) d \tau .
\end{array}
$$

Then we have

$$
\begin{aligned}
& \hat{g}_{\phi, \pm}^{(1)}(x, t)=\frac{1}{\sqrt{\pi}} r^{-(n-1) / 2} \exp \left\{-i \lambda_{c}(x, t) t \pm i \xi\left(x, \lambda_{c}(x, t)\right)\right\} \\
& \quad \times \int_{e_{1}} \exp \left\{ \pm \frac{i}{2}\left(\lambda-\lambda_{c}\right)^{2}\left(\partial_{\lambda}^{2} \xi\right)\left(x, \lambda_{c}\right)\right\} a_{ \pm}(x, t, \lambda) \exp \{ \pm i b(x, t, \lambda)\} d \lambda
\end{aligned}
$$

where

$$
\begin{aligned}
& a_{ \pm}(x, t, \lambda)=\omega\left(v(x, t)\left\{\lambda-\lambda_{c}(x, t)\right\}\right)\left\{\lambda-\eta(\lambda) V_{1}(x)\right\}^{-1 / 4} \zeta_{\phi, \pm}(x, \lambda) \\
& b(x, t, \lambda)=\frac{1}{2}\left\{\lambda-\lambda_{c}(x, t)\right\}^{3} \int_{0}^{1}(1-\tau)^{2}\left(\partial_{\lambda}^{3} \xi\right)\left(x, \lambda_{c}+\left(\lambda-\lambda_{c}\right) \tau\right) d \tau
\end{aligned}
$$

By (5.15) and (5.5) we have noting $v(x, t) \geq 1$,

$$
\begin{array}{ll}
\left|\partial_{\lambda}^{l} a_{ \pm}(x, t, \lambda)\right| \leq C_{21} v^{l}(x, t) r^{-1-\tilde{\delta}} & (l=0,1,2) \\
\left|\partial_{\lambda}^{l} b(x, t, \lambda)\right| \leq C_{21}\left|\lambda-\lambda_{c}(x, t)\right|^{3-l} r & (l=0,1,2) .
\end{array}
$$

We put

$$
h_{ \pm}(x, t, \lambda)=a_{ \pm}(x, t, \lambda) \exp \{ \pm i b(x, t, \lambda)\} .
$$

Then obviously,

$$
\begin{aligned}
& \partial_{\lambda} h_{ \pm}=\left\{\partial_{\lambda} a_{ \pm} \pm i a_{ \pm} \partial_{\lambda} b\right\} \exp \{ \pm i b\} \\
& \partial_{\lambda}^{2} h_{ \pm}=\left\{\partial_{\lambda}^{2} a_{ \pm} \pm 2 i \partial_{\lambda} a_{ \pm} \partial_{\lambda} b \pm i a_{ \pm} \partial_{\lambda}^{2} b-a_{ \pm}\left(\partial_{\lambda} b\right)^{2}\right\} \exp \{ \pm i b\}
\end{aligned}
$$

Lemma 5.5. Let $v(x, t)=r^{1 / 3}$ in (5.19). Then there exists a $C_{22}>0$ such that for any $x \in B\left(R_{2}+1\right)$ and $t_{2}(x) \leq \pm t \leq t_{1}(x)$,

$$
\left|\hat{g}_{\phi, \pm}^{(1)}(x, t)\right| \leq C_{22} r^{-(n-1) / 2} r^{-3 / 2-\tilde{\delta}} .
$$

Proof. Note that the support in $\lambda$ of $h_{ \pm}(x, t, \lambda)$ is contained in $e_{1}$. Then by use of the equality

$$
h_{ \pm}(x, t, \lambda)=h_{ \pm}\left(x, t, \lambda_{c}\right)+\left(\lambda-\lambda_{c}\right) \int_{0}^{1}\left(\partial_{\lambda} h_{ \pm}\right)\left(x, t, \lambda_{c}+\left(\lambda-\lambda_{c}\right) \tau\right) d \tau
$$

we have for any sufficiently large $N$, 


$$
\begin{aligned}
\int_{e_{1}} \exp \left\{ \pm 2^{-1} i\left(\lambda-\lambda_{c}\right)^{2}\left(\partial_{\lambda}^{2} \xi\right)\left(x, \lambda_{c}\right)\right\} h_{ \pm}(x, t, \lambda) d \lambda \\
=h_{ \pm}\left(x, t, \lambda_{c}\right) \int_{-N}^{N} \exp \left\{ \pm 2^{-1} i\left(\lambda-\lambda_{c}\right)^{2}\left(\partial_{\lambda}^{2} \xi\right)\left(x, \lambda_{c}\right)\right\} d \lambda \\
+\int_{-N}^{N} \exp \left\{ \pm 2^{-1} i\left(\lambda-\lambda_{c}\right)^{2}\left(\partial_{\lambda}^{2} \xi\right)\left(x, \lambda_{c}\right)\right\}\left(\lambda-\lambda_{c}\right) d \lambda \\
\times \int_{0}^{1}\left(\partial_{\lambda} h_{ \pm}\right)\left(x, t, \lambda_{c}+\left(\lambda-\lambda_{c}\right) \tau\right) d \tau .
\end{aligned}
$$

Here applying the Fresnel integral formula, we have

$$
\begin{gathered}
\lim _{N \rightarrow \infty} \int_{-N}^{N} \exp \left\{ \pm 2^{-1} i\left(\lambda-\lambda_{c}\right)^{2}\left(\partial_{\lambda}^{2} \xi\right)\left(x, \lambda_{c}\right)\right\} d \lambda \\
=\sqrt{2 \pi}\left|\left(\partial_{\lambda}^{2} \xi\right)\left(x, \lambda_{c}\right)\right|^{-1 / 2} \exp (\mp \pi i / 4) .
\end{gathered}
$$

On the other hand, since the Lebesgue theorem shows that

$$
\lim _{N \rightarrow \infty} \int_{0}^{1}\left(\partial_{\lambda} h_{ \pm}\right)\left(x, t, \lambda_{c}+\left( \pm N-\lambda_{c}\right) \tau\right) d \tau=0,
$$

integrating by parts and changing the order of integration, we have

$$
\begin{aligned}
\lim _{N \rightarrow \infty} \int_{-N}^{N} \exp \left\{ \pm 2^{-1} i\left(\lambda-\lambda_{c}\right)^{2}\left(\partial_{\lambda}^{2} \xi\right)\left(x, \lambda_{c}\right)\right\}\left(\lambda-\lambda_{c}\right) d \lambda \\
\quad \times \int_{0}^{1}\left(\partial_{\lambda} h_{ \pm}\right)\left(x, t, \lambda_{c}+\left(\lambda-\lambda_{c}\right) \tau\right) d \tau \\
= \pm i\left\{\left(\partial_{\lambda}^{2} \xi\right)\left(x, \lambda_{c}\right)\right\}^{-1} \int_{0}^{1} \tau d \tau \int_{\Sigma} \exp \left\{ \pm 2^{-1} i\left(\lambda-\lambda_{c}\right)^{2}\right. \\
\left.\quad \times\left(\partial_{\lambda}^{2} \zeta\right)\left(x, \lambda_{c}\right)\right\}\left(\partial_{\lambda}^{2} h_{ \pm}\right)\left(x, t, \lambda_{c}+\left(\lambda-\lambda_{c}\right) \tau\right) d \lambda,
\end{aligned}
$$

where

$$
\Sigma=\left\{\lambda ; \lambda_{3}-\lambda_{c} \leq\left(\lambda-\lambda_{c}\right) \tau \leq \lambda_{4}-\lambda_{c} \quad \text { and } \quad\left|\left(\lambda-\lambda_{c}\right) \tau\right| \leq v^{-1}\right\},
$$

if we note that $\omega(\lambda)=0$ for $|\lambda| \geq 1$ and $h_{ \pm}(x, t, \lambda)=0$ for $\lambda \notin e_{1}$. Taking account of (5.5), (5.23) and (5.24), we now have from (5.20), (5.27) and (5.29) - (5.31) the following inequalities which prove (5.28):

$$
\begin{aligned}
& \left|\hat{g}_{\phi, \pm}^{(1)}(x, t)\right| \leq C_{23} r^{-(n-1) / 2}\left[r^{-1-\tilde{\delta}} r^{-1 / 2}+r^{-1} \int_{0}^{1} \tau d \tau\right. \\
& \left.\quad \times \int_{\left|\lambda-\lambda_{c}\right|<(v \tau)^{-1}}\left\{v^{2}+v r\left|\lambda-\lambda_{c}\right|^{2} \tau^{2}+r\left|\lambda-\lambda_{c}\right| \tau+r^{2}\left|\lambda-\lambda_{c}\right|^{4} \tau^{4}\right\} r^{-1-\tilde{\delta}} d \lambda\right] \\
& =C_{23} r^{-(n-1) / 2} r^{-1-\tilde{\delta}}\left[r^{-1 / 2}+r^{-1} \int_{0}^{1} 2 \tau\left\{v \tau^{-1}+\frac{1}{3} r v^{-2} \tau^{-1}\right.\right. \\
& \left.\quad+\frac{1}{2} r v^{2} \tau^{-1}+\frac{1}{5} r^{2} v^{5} \tau^{-1}\right\} d \tau \mid
\end{aligned}
$$




$$
\begin{aligned}
& =C_{23} r^{-(n-1) / 2} r^{-1-\tilde{\delta}}\left[r^{-1 / 2}+r^{-1}\left\{2 v+\frac{5}{3} r v^{-2}+\frac{2}{5} r^{2} v^{-5}\right\}\right] \\
& =C_{23} r^{-(n-1) / 2} r^{-1-\tilde{\delta}}\left(r^{-1 / 2}+\frac{61}{15} r^{-2 / 3}\right)
\end{aligned}
$$

where in the last equality we have used $v=r^{1 / 3}$.

q.e.d.

Lemma 5.6. Let $v(x, t)$ be as in the above lemma. Then there exists a $C_{24}>0$ such that for any $x \in B\left(R_{2}+1\right)$ and $t_{2}(x) \leq \pm t \leq t_{1}(x)$,

$$
\left|\hat{g}_{\phi, \pm}^{(2)}(x, t)\right| \leq C_{24} r^{-(n-1) / 2} r^{-5 / 3-\tilde{\delta}}
$$

Proof. We put

$$
d_{ \pm}(x, t, \lambda)=\left\{1-\omega\left(v(x, t)\left\{\lambda-\lambda_{c}(x, t)\right\}\right)\right\}\left\{\lambda-\eta(\lambda) V_{1}(x)\right\}^{-1 / 4} \zeta_{\phi \pm}(x, \lambda) .
$$

Then it follows from (5.15) that

$$
\left|\partial_{\lambda}^{l} d_{ \pm}(x, t, \lambda)\right| \leq C_{25} v^{l}(x, t) r^{-1-\tilde{\delta}} \quad(l=0,1,2)
$$

in the whole $e_{1}$. Note that $d_{ \pm}(x, t, \lambda)=0$ in $\left\{\lambda \in e_{1} ;\left|\lambda-\lambda_{c}\right|<(2 v)^{-1}\right\}$. On the other hand, it follows from (5.16) and (5.4) that for any $\lambda \in e_{1}$ satisfying $\left|\lambda-\lambda_{c}(x, t)\right| \geq(2 v)^{-1}$,

$$
\begin{aligned}
\left|t \mp \partial_{\lambda} \xi(x, \lambda)\right| & =\left|\partial_{\lambda} \xi\left(x, \lambda_{c}\right)-\partial_{\lambda} \xi(x, \lambda)\right| \\
& =\left|\lambda-\lambda_{c}\right|\left|\left(\partial_{\lambda}^{2} \xi\right)\left(x, \lambda_{c}+\left(\lambda-\lambda_{c}\right) \theta\right)\right| \quad(0<\theta<1) \\
& \geq(2 v)^{-1} C_{17}^{-1} r=\left(2 C_{17}\right)^{-1} r^{2 / 3} .
\end{aligned}
$$

Now, integrating by parts gives

$$
\begin{aligned}
& \sqrt{\pi} r^{(n-1) / 2} \hat{g}_{\phi, \pm}^{(2)}(x, t)=\int_{e_{1}} \exp \{-i \lambda t \pm i \xi(x, \lambda)\} d_{ \pm}(x, t, \lambda) d \lambda \\
& =-\int_{e_{1}} \exp \{-i \lambda t \pm i \xi(x, \lambda)\}\left(t \mp \partial_{\lambda} \xi\right)^{-2}\left[\partial_{\lambda}^{2} d_{ \pm} \pm 3 \partial_{\lambda}^{2} \xi\left(t \mp \partial_{\lambda} \xi\right)^{-1} \partial_{\lambda} d_{ \pm}\right. \\
& \left.\quad+\left\{3\left(\partial_{\lambda}^{2} \xi\right)^{2}\left(t \mp \partial_{\lambda} \xi\right)^{-2} \pm \partial_{\lambda}^{3} \xi\left(t \mp \partial_{\lambda} \xi\right)^{-1}\right\} d_{ \pm}\right] d \lambda .
\end{aligned}
$$

So, applying (5.34) and (5.35) in the right side of (5.36), we obtain

$$
\left|\hat{g}_{\phi, \pm}^{(2)}(x, t)\right| \leq C_{26} r^{-(n-1) / 2} r^{-4 / 3} r^{-1-\tilde{\delta}}\left\{v^{2}+r r^{-2 / 3} v+\left(r^{2} r^{-4 / 3}+r r^{-2 / 3}\right)\right\},
$$

which proves (5.32) since $v=r^{1 / 3}$.

q.e.d.

Proof of Proposition 4.1. Let $\mu$ be a constant satisfying $0<\mu<2 \tilde{\delta}$. Then we have

$$
\begin{aligned}
& \left\{ \pm \int_{0}^{ \pm \infty}\left\|\hat{g}_{\phi, \pm}(\cdot, t)\right\| d t\right\}^{2} \\
& \quad \leq \int_{0}^{ \pm \infty}(1+|t|)^{-1-\mu} d t\left\{\int_{0}^{ \pm \infty}(1+|t|)^{1+\mu} d t \int_{B\left(R_{2}+1\right)}\left|\hat{g}_{\phi, \pm}(x, t)\right|^{2} d x\right\}
\end{aligned}
$$




$$
= \pm \mu^{-1} \int_{B\left(R_{2}+1\right)} d x \int_{0}^{ \pm \infty}\left|\hat{g}_{\phi, \pm}(x, t)\right|^{2}(1+|t|)^{1+\mu} d t .
$$

We divide the integrand of the right side as follows:

$$
\begin{aligned}
& \pm \int_{0}^{ \pm \infty}\left|\hat{g}_{\phi, \pm}(x, t)\right|^{2}(1+|t|)^{1+\mu} d t \\
& \quad= \pm\left[\int_{0}^{ \pm t_{2}(x)}+\int_{ \pm t_{2}(x)}^{ \pm t_{1}(x)}+\int_{ \pm t_{1}(x)}^{ \pm \infty}\right]\left|\hat{g}_{\phi, \pm}(x, t)\right|^{2}(1+|t|)^{1+\mu} d t \\
& \quad=I_{1}+I_{2}+I_{3} .
\end{aligned}
$$

By (5.14) of Lemma 5.3 we have

$$
\begin{aligned}
& I_{1} \leq \pm 2 C_{19}^{2} r^{-(n-1)} r^{-2-2 \delta} \int_{0}^{ \pm t_{2}(x)}(1+|t|)^{1+\mu}\left(t_{4}(x)-|t|\right)^{-4} \\
& \times\left[1+r^{4}\left(t_{4}(x)-|t|\right)^{-4}\right] d t .
\end{aligned}
$$

Thus, it follows from (5.10) and (5.8) that

$$
\begin{aligned}
I_{1} & \leq 2(2+\mu)^{-1} C_{19}^{2} C_{18}^{4} r^{-(n-1)} r^{-2-2 \tilde{\delta}} r^{-4}\left(1+C_{17} r^{2}\right)^{2+\mu}\left(1+C_{18}^{4}\right) \\
& \leq C_{27} r^{-(n-1)} r^{-4-2 \tilde{\delta}+\mu} .
\end{aligned}
$$

By (5.13) of Lemma 5.3 we have

$$
\begin{aligned}
I_{3} \leq \pm 2 C_{19}^{2} r^{-(n-1)} r^{-2-2 \tilde{\delta}} \int_{ \pm t_{1}(x)}^{ \pm \infty}(1+|t|)^{1+\mu}\left(|t|-t_{3}(x)\right)^{-4} \\
\quad \times\left[1+r^{4}\left(|t|-t_{3}(x)\right)^{-4}\right] d t \\
\leq \pm 2 C_{19}^{2} r^{-(n-1)} r^{-2-2 \tilde{\delta}} \int_{ \pm t_{1}(x)}^{ \pm \infty} 2^{\mu}\left\{\left(|t|-t_{3}(x)\right)^{1+\mu}+\left(t_{3}(x)+1\right)^{1+\mu}\right\} \\
\times\left(|t|-t_{3}(x)\right)^{-4}\left[1+r^{4}\left(|t|-t_{3}(x)\right)^{-4}\right] d t .
\end{aligned}
$$

Thus, it follows from (5.9) and (5.8) that

$$
\begin{aligned}
I_{3} & \leq 2^{1+\mu} C_{19}^{2} r^{-(n-1)} r^{-2-2 \tilde{\delta}}\left\{(2-\mu)^{-1}\left(C_{18} r\right)^{-2+\mu}+3^{-1}\left(1+C_{17} r\right)^{1+\mu}\right. \\
& \left.\times\left(C_{18} r\right)^{-3}\right\}\left(1+C_{18}^{4}\right) \\
& \leq C_{28} r^{-(n-1)} r^{-4-2 \tilde{\delta}+\mu} .
\end{aligned}
$$

Further, by Lemmas 5.5 and 5.6,

$$
\begin{aligned}
I_{2} & \leq \pm 2 \int_{ \pm t_{2}(x)}^{ \pm t_{1}(x)}(1+|t|)^{1+\mu}\left\{\left|\hat{g}_{\phi, \pm}^{(1)}(x, t)\right|^{2}+\left|\hat{g}_{\phi, \pm}^{(2)}(x, t)\right|^{2}\right\} d t \\
& \leq 2(2+\mu)^{-1} r^{-(n-1)}\left(C_{22}^{2} r^{-3-2 \tilde{\delta}}+C_{24}^{2} r^{-10 / 3-2 \tilde{\delta}}\right)\left(1+t_{1}(x)\right)^{2+\mu}
\end{aligned}
$$

Thus, it follows from (5.8) that

$$
I_{2} \leq C_{29} r^{-(n-1)} r^{-1-2 \tilde{\delta}+\mu}
$$

Summarizing these inequalities, we have from (5.37) and (5.38), 


$$
\begin{aligned}
\{ & \left. \pm \int_{0}^{ \pm \infty}\left\|\hat{g}_{\phi, \pm}(\cdot, t)\right\| d t\right\}^{2} \\
& \leq \mu^{-1} \int_{B\left(R_{2}+1\right)}\left\{\left(C_{27}+C_{28}\right) r^{-4-2 \tilde{\delta}+\mu}+C_{29} r^{-1-2 \tilde{\delta}+\mu}\right\} r^{-(n-1)} d x<\infty .
\end{aligned}
$$

Thus, (4.5) holds and the proof of Proposition 4.1 is complete.

q.e.d.

\section{References}

[1] Alsholm, P. and Kato, T., Scattering with long-range potentials, Proc. Symp. Pure Math., 23 (1973), 393-399.

[2] Amrein, W. O., Martin, Ph. A. and Misra, B., On the asymptotic condition of scattering theory, Helv. Phys. Acta, 43 (1970), 313-344.

[ 3 ] Asada, K. and Fujiwara, D., On some oscillatory integral transformations in $L^{2}\left(\boldsymbol{R}^{n}\right)$, Japan J. Math., 4 (1978), 299-361.

[4] Ben-Artzi, M. and Devinatz, A., Spectral and scattering for the adiabatic oscillator and related potentials, J. Math. Phys., 20 (1979), 594-607.

[ 5 ] Buslaev, V. S. and Matveev, V. B., Wave operators for the Schrödinger equation with a slowly decreasing potential, Theor. Math. Phys., 2 (1970), 266-274 (English trans. from Russian).

[6] Calderón, A. P. and Vaillancourt, R., A class of bounded pseudo-differential operators, Proc. Nat. Acad. Sci. USA, 69 (1972), 1185-1187.

[ 7 ] Dollard, J. D., Asymptotic convergence and the Coulomb interaction, J. Math. Phys., 5 (1964), 729-738.

[ 8 ] Dollard, J. D. and Friedman, C. N., Existence of the Møller wave operators for $V(r)=$ $\lambda \sin \left(\mu r^{\alpha}\right) / r^{\beta}$, Ann. Phys., 111 (1978), 251-266.

[9] Hörmander, L., The existence of wave operators in scattering theory, Math. Z., 146 (1976), 69-91.

[10] Ikebe, T. and Isozaki, H., A stationary approach to the completeness of long-range wave operators (preprint 1980).

[11] Kako, T., Existence and equivalence of two types of long-range modified wave operators, J. Fac. Sci. Univ. Tokyo Sec. IA, 25 (1978), 133-147.

[12] Kato, T., Scattering theory with two Hilbert spaces, J. Func. Anal., 1 (1967), 342-369.

[13] Kitada, H., Scattering theory for Schrödinger operators with long-range potentials, II, J. Math. Soc. Japan, 30 (1978), 603-632.

[14] Mochizuki, K. and Uchiyama, J., Radiation conditions and spectral theory for 2-body Schrödinger operators with "oscillating" long-range potentials, I, J. Math. Kyoto Univ., 18 (1978), 377-408.

[15] — Radiation conditions and spectral theory for 2-body Schrödinger operators with "oscillating" long-range potentials, II, J. Math. Kyoto Univ., 19 (1979), 47-70.

[16] Yosida, K., Functional analysis, Berlin-Göttingen-Heidelberg, Springer 1965. 